University of Warwick institutional repository: http://go.warwick.ac.uk/wrap This paper is made available online in accordance with publisher policies. Please scroll down to view the document itself. Please refer to the repository record for this item and our policy information available from the repository home page for further information.

To see the final version of this paper please visit the publisher's website. Access to the published version may require a subscription.

Author(s): Oliver Jenkinson, Luis Felipe Gonzalez and Mariusz Urbanski Article Title: On Transfer Operators for Continued Fractions with Restricted Digits

Year of publication: 2003

Link to published version: http://dx.doi.org/10.1112/S0024611502013904 Publisher statement: None 


\title{
ON TRANSFER OPERATORS FOR CONTINUED FRACTIONS WITH RESTRICTED DIGITS
}

\author{
OLIVER JENKINSON, LUIS FELIPE GONZALEZ \\ AND MARIUSZ URBAŃSKI
}

\section{Introduction}

For a non-empty subset $\mathscr{I} \subset \mathbb{N}$, let $\Lambda_{\mathscr{I}}$ denote the set of real numbers $x \in[0,1]$ for which the infinite continued fraction expansion

$$
x=\frac{1}{a_{1}+\frac{1}{a_{2}+\ldots}}
$$

has each partial quotient $a_{i}$ belonging to the alphabet $\mathscr{I}$. Let $T:[0,1] \rightarrow[0,1]$ be the continued fraction (or Gauss) map, given by $T(x)=1 / x(\bmod 1)$. The set $\Lambda_{\mathscr{I}}$ is invariant under $T$.

When the alphabet $\mathscr{I}$ is finite, the irrationals in $\Lambda_{\mathscr{I}}$ are all badly approximable by rationals (cf. [44]). The arithmetic properties of such $\Lambda_{\mathscr{I}}$ have therefore been widely studied, with particular emphasis on the set $E_{2}$, corresponding to the choice $\mathscr{I}=\{1,2\}$ (cf. $[\mathbf{1 6}, \mathbf{8}, \mathbf{2 2}]$ ). The Hausdorff dimension of sets such as $E_{2}$ yields insight into various problems in Diophantine approximation, particularly in connection with the Markoff and Lagrange spectra (cf. [8, 13, 24]). Further geometric measure-theoretic properties have been studied by Mauldin and Urbański [31], who extended this investigation to the case of infinite alphabets $\mathscr{I}$ as part of a wider analysis of infinite conformal iterated function systems [30].

A powerful approach to these problems is provided by the thermodynamic formalism of Ruelle [39]. To a continuous function $g:[0,1] \rightarrow \mathbb{R}$, we associate the Ruelle transfer operator $L_{\mathscr{I}, g}$, defined by the formula

$$
L_{\mathscr{I}, g} f(x)=\sum_{n \in \mathscr{I}} \exp \left[g\left(\frac{1}{x+n}\right)\right] f\left(\frac{1}{x+n}\right),
$$

whenever this sum is convergent.

The choice $g(x)=g_{\beta}(x)=-\beta \log \left|T^{\prime}(x)\right|=\log x^{2 \beta}$, for a real parameter $\beta$, is of particular importance, leading to the formula

$$
\mathscr{L}_{\mathscr{I}, \beta} f(z)=L_{\mathscr{I}, g_{\beta}} f(z)=\sum_{n \in \mathscr{I}}\left(\frac{1}{z+n}\right)^{2 \beta} f\left(\frac{1}{z+n}\right),
$$

where again if $\mathscr{I}$ is infinite then care is needed in the choice of $\beta$ and $f$ to ensure

Received 25 October 2001; revised 8 April 2002.

2000 Mathematics Subject Classification 37D35, 37D20, 30 B70.

The first author acknowledges the warm hospitality of the University of North Texas, where some of this research took place.

The second author was supported by the CONACYT grant no. 110864/110990.

The third author was supported in part by the NSF Grant no. DMS 0100078. 
convergence of the sum. Specifically, we suppose that $\operatorname{Re}(\beta)>\theta_{\mathscr{I}}$, where $\operatorname{Re}(\beta)=\theta_{\mathscr{I}}$ is the abscissa of convergence of the series $\sum_{n \in \mathscr{I}} n^{-2 \beta}$, and that $f \in C^{0}[0,1]$. In what follows, we will consider this operator acting on functions $f$ that are at least $C^{\infty}$, and this will additionally ensure that it has countable spectrum (see $\S 3$ ).

One of the reasons for the importance of $\mathscr{L}_{\mathscr{I}, \beta}$ is its relation to Hausdorff dimension. Under appropriate hypotheses on $\mathscr{I}$ (see $\S 2$ ) there is a unique real value of $\beta$ for which $\mathscr{L}_{\mathscr{I}, \beta}$ has leading eigenvalue equal to 1 , and this value is precisely the Hausdorff dimension of $\Lambda_{\mathscr{I}}$ (see [7, 30]). This characterisation has been used to approximate the Hausdorff dimension of $\Lambda_{\{1,2\}}$ (see $\left.[\mathbf{8}, \mathbf{2 2}, \mathbf{2 4}]\right)$. The eigenfunction of this leading eigenvalue is, suitably normalised, the density function for the natural geometric $T$-invariant probability measure supported on $\Lambda_{\mathscr{I}}$. For $\mathscr{I}=\mathbb{N}$ this density is $(1 / \log 2)(1 /(1+x))$, corresponding to the wellknown Gauss measure. The second eigenvalue of $\mathscr{L}_{\mathscr{I}, \beta}$ determines the rate of correlation decay for the dynamical system $T: \Lambda_{\mathscr{I}} \rightarrow \Lambda_{\mathscr{I}}$ with respect to the geometric invariant measure; for $\mathscr{I}=\mathbb{N}$ this corresponds to Wirsing's constant (cf. $[14,34,51])$. It can also be related to the efficiency of numerical algorithms for approximating the Hausdorff dimension of $\Lambda_{\mathscr{I}}$ (cf. [21]). The lower-lying eigenvalues (the so-called Ruelle resonances, cf. $[\mathbf{4 0}, \mathbf{4 1}]$ ) determine finer mixing properties of $T: \Lambda_{\mathscr{I}} \rightarrow \Lambda_{\mathscr{I}}$.

It is conjectured that the full $C^{\infty}$ spectrum of various Ruelle transfer operators associated to expanding dynamical systems (including, for example, the linearised Feigenbaum renormalisation operator [11]) is real. Empirical evidence supports these conjectures, although a satisfactory explanation for the phenomenon is lacking. If the underlying expanding map is piecewise linear, then it is easily proved that the spectrum is real. For non-linear expanding maps where one branch dominates the other in a certain sense, Rugh [43] has shown that the spectrum of certain associated transfer operators is indeed real. However, reality of the spectrum is not ubiquitous, as demonstrated by Levin [29].

A remarkable analysis of Babenko [3] established the reality of the spectrum of $\mathscr{L}_{\mathbb{N}, 1}$, the Perron-Frobenius operator for the Gauss map on $[0,1]$. His method is very different from the perturbative approach of Rugh, hinging instead on an algebraization of the problem by means of an integral transform. This analysis of the Perron-Frobenius operator was further elaborated by Mayer and Roepstorff $[34,35]$, while a significant generalisation of Babenko's theorem to the family $\mathscr{L}_{\mathbb{N}, \beta}$, with $\beta>\frac{1}{2}$, was given by Mayer [33]. The main purpose of our article is to further develop this algebraization program. It will be shown that the transfer operators $\mathscr{L}_{\mathscr{I}, \beta}$ associated to general invariant sets $\Lambda_{\mathscr{I}}$ may also be transformed in such a way that the reality of their spectra is readily apparent. These operators will act primarily on canonical spaces such as $C^{\infty}[0,1]$ and $C^{\omega}[0,1]$. For example we prove the following.

TheOREM. Suppose $\mathscr{I}$ is a non-empty subset of $\mathbb{N}$, and $\beta>\max \left(0, \theta_{\mathscr{I}}\right)$. Then the operator $\mathscr{L}_{\mathscr{I}, \beta}: C^{\infty}[0,1] \rightarrow C^{\infty}[0,1]$ has real spectrum. This spectrum is a sequence of real numbers union its accumulation point at 0 .

This result will follow from Theorems 2 and 3, in $\S \S 5$ and 6 respectively, where identical spectral properties are proved for $\mathscr{L}_{\mathscr{I}, \beta}$ acting on the nuclear space $C^{\omega}[0,1]$ and on various spaces of holomorphic functions. The scheme of proof is as follows. To each alphabet $\mathscr{I}$ we will associate a theta function 
$\vartheta_{\mathscr{I}}(s)=\sum_{n \in \mathscr{I}} e^{-n s}$, a Stieltjes measure $d m_{\mathscr{I}}(s)=\vartheta_{\mathscr{I}}(s) d s$, and an integral transform given by $\mathscr{T}_{\mathscr{I}, \beta} \varphi(z)=\int_{0}^{\infty} s^{\beta-1 / 2} e^{-s z} \varphi(s) d m_{\mathscr{I}}(s)$. The transfer operator will be shown to preserve a Hilbert space $\mathscr{H}_{\mathscr{I}, \beta}=\mathscr{T}_{\mathscr{I}, \beta}\left(L^{2}\left(m_{\mathscr{I}}\right)\right)$ of holomorphic functions, on which its action is similar to a selfadjoint integral operator on $L^{2}\left(m_{\mathscr{I}}\right)$. It follows that $\mathscr{L}_{\mathscr{I}, \beta}: \mathscr{H}_{\mathscr{I}, \beta} \rightarrow \mathscr{H}_{\mathscr{I}, \beta}$ has real spectrum. Since it can be shown (Lemma 6) that $\mathscr{H}_{\mathscr{I}, \beta}$ is densely and continuously embedded in $C^{\infty}[0,1]$, a recent intertwining theorem of Bandtlow [4] implies that the spectrum of $\mathscr{L}_{\mathscr{I}, \beta}$ on these two spaces is the same, thus establishing the above theorem. In the same way it will be shown that the non-essential spectrum of $\mathscr{L}_{\mathscr{I}, \beta}$ acting on Hölder function spaces, or on spaces of $C^{k}$ functions, is also real.

In the second part of this article we consider the analytic dependence of $\mathscr{L}_{\mathscr{Y}, \beta}$ on the parameter $\beta$. If $\beta \in \mathbb{C}$, with $\operatorname{Re}(\beta)>\theta_{\mathscr{I}}$, then $\mathscr{L}_{\mathscr{I}, \beta}$ is clearly well defined. For such $\beta$ it can again be shown (Theorem 1) that $\mathscr{L}_{\mathscr{I}, \beta}$ is similar to an (in general non-selfadjoint) integral operator. (If $\mathscr{I}$ is finite, so that (2) is convergent for all $\beta \in \mathbb{C}$, this result requires the assumption $\operatorname{Re}(\beta)>0$.)

We consider $\mathscr{L}_{\mathscr{I}, \beta}$ as an element of the complex Banach space of bounded linear operators on a suitable Hilbert space. This ensures a canonical notion of analyticity for the map $\beta \mapsto \mathscr{L}_{\mathscr{I}, \beta}$, equivalent to complex differentiability. The analyticity of this map in the half-plane $\operatorname{Re}(\beta)>\theta_{\mathscr{I}}$ suggests the possibility of an analytic continuation to a wider domain in the $\beta$-plane. The original result in this direction is due to Mayer [33], who analysed the case $\mathscr{I}=\mathbb{N}$. Using a somewhat weaker notion of analyticity, he proved that $\beta \mapsto \mathscr{L}_{\mathbb{N}, \beta}$ has a meromorphic continuation to the whole $\beta$-plane, the only poles being simple ones at $\beta=\frac{1}{2}(1-k)$, with $k \in \mathbb{Z}_{\geqslant 0}$.

The situation for general alphabets $\mathscr{I}^{2}$ is rather more recondite. The simplest situation is when $\mathscr{I}$ is finite; in this case $\theta_{\mathscr{I}}=-\infty$, and the analytic continuation, in this case to the entire $\beta$-plane, is immediate. When $\mathscr{I}$ is infinite then $\beta=\theta_{\mathscr{I}}$ is a non-removable singularity, and in general there is no analytic continuation past this point. However, for certain infinite alphabets $\mathscr{I}$, a meromorphic continuation does exist, as described in Theorem 5. For example, if $\mathscr{I}$ is an arithmetic progression $\{a+b n\}$, a monomial sequence $\left\{n^{b}\right\}$, or a geometric progression $\left\{b^{n}\right\}$, then there is a meromorphic continuation to the whole complex $\beta$-plane. If $\mathscr{I}$ is either the set of all prime numbers, or the set of numbers which are the sum of two squares, then $\theta_{\mathscr{I}}=\frac{1}{2}$. In both these cases an analytic continuation past $\beta=\theta_{\mathscr{I}}$ is possible, but there is no meromorphic extension to the whole plane. If $\mathscr{I}$ is a sequence of super-exponential growth, then $\theta_{\mathscr{I}}=0$, and the line $\operatorname{Re}(\beta)=0$ is a natural boundary, so that no analytic continuation is possible.

The article is organised as follows. In $\S 2$ we introduce the fundamental objects necessary for our analysis. The action of $\mathscr{L}_{\mathscr{I}, \beta}$ on various function spaces is discussed in $\S 3$. In $\S 4$ we develop the basic properties of the integral operator and the integral transform. The conjugacy of $\mathscr{L}_{\mathscr{I}, \beta}$ with the integral operator is established in $\S 5$, as is the reality of the spectrum for $\beta$ real. In $\S 6$ this spectral property is extended to various other function spaces. In $\S 7$ we consider the analytic properties of the map $\beta \mapsto \mathscr{L}_{\mathscr{I}, \beta}$. This is related (Theorem 4) to the analytic properties of a certain Hurwitz zeta function, which then yields precise results (Theorem 5) for certain alphabets $\mathscr{I}$ of an arithmetic nature.

Acknowledgements. This paper has greatly benefited from various enlightening discussions with Oscar Bandtlow on some fine points of functional analysis. We are also grateful to Richard Sharp for some helpful comments on Theorem 5(vii). 


\section{The fundamental objects}

A non-empty subset $\mathscr{I}$ of $\mathbb{N}$ will be called an alphabet. We associate to $\mathscr{I}$ the following objects:

the limit set $\Lambda_{\mathscr{I}}$, defined as the set of real numbers in $[0,1]$ whose continued fraction partial quotients all belong to $\mathscr{I}$;

the Hausdorff dimension $\delta_{\mathscr{I}}$ of the limit set $\Lambda_{\mathscr{I}}$;

the finiteness parameter $\theta_{\mathscr{I}}$, defined as the infimum of those real numbers $\theta$ for which

$$
\sum_{n \in \mathscr{I}} \frac{1}{n^{2 \beta}}
$$

converges for all $\beta \in \mathbb{C}$ with $\operatorname{Re}(\beta)>\theta$.

If $\mathscr{I}=\mathbb{N}$ then $\Lambda_{\mathscr{I}}$ is the set of irrationals in $[0,1]$, and in general when $\mathscr{I}$ is infinite $\Lambda_{\mathscr{I}}$ is homeomorphic to the irrationals (in particular, it is not compact). If $\mathscr{I}$ is finite with cardinality greater than 1 , then $\Lambda_{\mathscr{I}}$ is a Cantor subset of $[0,1]$. Moreover, we have $0<\delta_{\mathscr{I}}<1$ unless $\mathscr{I}=\mathbb{N}$ (when the dimension is 1 ) or $\mathscr{I}$ is a singleton (when the dimension is 0 ).

If $\mathscr{I}$ is finite then clearly $\theta_{\mathscr{I}}=-\infty$. If $\mathscr{I}$ is infinite then $\theta_{\mathscr{I}} \in\left[0, \frac{1}{2}\right]$, and the series (3) has a singularity at $\beta=\theta_{\mathscr{I}}$ since it is a Dirichlet series all of whose coefficients are non-negative (cf. [2, Theorem 11.13]).

To the alphabet $\mathscr{I}$ we further associate the following objects:

$N_{\mathscr{I}}:=\min \mathscr{I}$;

the zeta function $\zeta_{\mathscr{I}}(s)=\sum_{n \in \mathscr{I}} n^{-s}$, defined initially for $\operatorname{Re}(s)>2 \theta_{\mathscr{I}}$, but in some cases admitting an analytic continuation to a larger domain in the $s$-plane;

the Hurwitz zeta function $\zeta_{\mathscr{I}}(s, z)=\sum_{n \in \mathscr{I}}(n+z)^{-s}$, defined initially for $z \in \mathbb{C} \backslash\left(-\infty,-N_{\mathscr{I}}\right], \operatorname{Re}(s)>2 \theta_{\mathscr{I}}$, but in some cases admitting an analytic continuation to a larger domain in the $s$-plane;

the theta function $\vartheta_{\mathscr{I}}(t)=\sum_{n \in \mathscr{I}} e^{-n t}$, defined for $t \in \mathbb{R}^{+}$;

the theta measure $m_{\mathscr{I}}$ on $\mathbb{R}^{+}$, defined by $d m_{\mathscr{I}}(t)=\vartheta_{\mathscr{I}}(t) d t$;

the right half-plane $R_{\mathscr{I}}=\left\{z \in \mathbb{C}: \operatorname{Re}(z)>-\frac{1}{2} N_{\mathscr{I}}\right\}$.

The nomenclature is motivated by that used in classical analytic number theory, in spectral geometry (for example, $[9,10,49]$ ), and in recent generalisations of Jorgenson and Lang (for example, see $[\mathbf{2 5}, \mathbf{2 6}]$ ). For instance, if $\mathscr{I}=\mathbb{N}$ then $\zeta_{\mathscr{I}}$ is Riemann's zeta function, and the theta function is simply $\theta_{\mathscr{I}}(t)=1 /\left(e^{t}-1\right)$. (Note, however, that the classical Hurwitz zeta function is defined by a summation over all non-negative (rather than positive) integers (cf. [6, p.502]). In this way our convention differs from the classical one.)

For any $\beta \in \mathbb{C}$, the kernel function $G_{\beta}: \mathbb{R}^{+} \times \mathbb{R}^{+} \rightarrow \mathbb{C}$ is defined by

$$
G_{\beta}(s, t)=\sum_{k=0}^{\infty} \frac{(-1)^{k}(s t)^{k+\beta-1 / 2}}{k ! \Gamma(k+2 \beta)} .
$$

In fact this kernel function is related to a Bessel function of the first kind (cf. [50, p. 40]) by $G_{\beta}(s, t)=\mathscr{J}_{2 \beta-1}(2 \sqrt{t s})$. The most important property of $G_{\beta}$ is that if $\beta \in \mathbb{R}$ then $G_{\beta}(s, t)=\overline{G_{\beta}(t, s)}$, so that any associated integral operator is selfadjoint.

For $\operatorname{Re}(\beta)>\theta_{\mathscr{I}}$ we associate to the pair $(\mathscr{I}, \beta)$ the following objects. 
The integral transform $\mathscr{T}_{\mathscr{I}, \beta}$ defined by

$$
\mathscr{T}_{\mathscr{I}, \beta} \varphi(z)=\int_{0}^{\infty} s^{\beta-1 / 2} e^{-s z} \varphi(s) d m_{\mathscr{I}}(s) .
$$

The space of integral transforms $\mathscr{H}_{\mathscr{I}, \beta}=\mathscr{T}_{\mathscr{I}, \beta}\left(L^{2}\left(m_{\mathscr{I}}\right)\right)$. This is a Hilbert space, its inner product inherited from $L^{2}\left(m_{\mathscr{I}}\right)$ via the map $\mathscr{T}_{\mathscr{I}, \beta}$, which is easily seen to be injective and which we declare to be a Hilbert space isomorphism. It will be shown that each $f \in \mathscr{H}_{\mathscr{I}, \beta}$ is a function analytic in the right half-plane $R_{\mathscr{I}}$.

The integral operator $\mathscr{K}_{\mathscr{I}, \beta}$ defined by

$$
\mathscr{K}_{\mathscr{I}, \beta} \varphi(t)=\int_{0}^{\infty} G_{\beta}(s, t) \varphi(s) d m_{\mathscr{I}}(s) .
$$

The transfer operator $\mathscr{L}_{\mathscr{I}, \beta}$ defined by

$$
\mathscr{L}_{\mathscr{I}, \beta} f(z)=\sum_{n \in \mathscr{I}}\left(\frac{1}{z+n}\right)^{2 \beta} f\left(\frac{1}{z+n}\right) .
$$

The main focus of our attention is the transfer operator $\mathscr{L}_{\mathscr{I}, \beta}$. As mentioned in $\S 1$, one of the reasons for this interest is its relation with Hausdorff dimension $\delta_{\mathscr{I}}$. If $\zeta_{\mathscr{I}}\left(2 \theta_{\mathscr{I}}\right)=\infty$ (in which case we say that $\mathscr{I}$ is regular, as in $[\mathbf{3 0}, \mathbf{3 1}$, where examples of irregular sets can also be found) then there is a unique real value of $\beta$ for which $\mathscr{L}_{\mathscr{I}, \beta}$ has spectral radius 1 , and this value is precisely $\delta_{\mathscr{I}}$ (see $[7,30])$. The transfer operator will be studied by means of the various auxiliary objects we have introduced. The integral transform $\mathscr{T}_{\mathscr{f}, \beta}$ resembles a Laplace-Mellin transform (cf. [25, p. 9]), although the exponent $\beta-\frac{1}{2}$ is carefully chosen for our purposes (cf. Remark 3). The reason for the choice of kernel function $G_{\beta}$ will become apparent in $\S 5$.

\section{Trace class transfer operators}

As is well known, the spectral properties of the transfer operator $\mathscr{L}_{\mathscr{I}, \beta}$ depend strongly upon the space on which it acts. In fact it will be convenient for $\mathscr{L}_{\mathscr{I}, \beta}$ to act on several different spaces, which we now specify.

For $0<\alpha \leqslant 1$, let $C^{\alpha}[0,1]$ denote the space of complex-valued $\alpha$-Hölder functions on $[0,1]$, equipped with its Banach norm. For real $\beta>\max \left(0, \theta_{\mathscr{I}}\right)$, the operator $\mathscr{L}_{\mathscr{I}, \beta}: C^{\alpha}[0,1] \rightarrow C^{\alpha}[0,1]$ enjoys various Perron-Frobenius-type properties. In particular, it has an essential spectral radius strictly smaller than its spectral radius (see $[\mathbf{4 2}])$.

Since $z \mapsto(z+n)^{-2 \beta}$ extends as a $C^{\omega}$ function to the unit interval, the operator $\mathscr{L}_{\mathscr{I}, \beta}$ in fact preserves various subspaces of $C^{\alpha}[0,1]$, notably the Banach spaces $C^{k}[0,1]$ for $k \in \mathbb{N}$, the Fréchet space $C^{\infty}[0,1]$, and the nuclear space $C^{\omega}[0,1]$. When acting on $C^{k}[0,1], \mathscr{L}_{\mathscr{T}, \beta}$ is again quasicompact, although its essential spectral radius shrinks to 0 as $k \rightarrow \infty$ (see [42]). In Proposition 2 it will be shown that the spectrum of $\mathscr{L}_{\mathscr{I}, \beta}$ on both $C^{\infty}[0,1]$ and $C^{\omega}[0,1]$ is a countable set accumulating at 0 .

The operator $\mathscr{L}_{\mathscr{J}, \beta}$ also preserves various spaces of holomorphic functions, and there is considerable freedom in selecting such spaces. For example, suppose that $D$ is a complex domain satisfying

(i) $D$ does not intersect $-\mathscr{I}$, 
(ii) $\bar{D} \subset \operatorname{int}\left(\bigcap_{n \in \mathscr{I}} S_{n}(D)\right)$, where $S_{n}(z):=(1 / z)-n$,

(iii) $D$ is bounded and simply connected.

There is an abundance of such domains $D$. For example, $D=\left\{z \in \mathbb{C}:|z-1|<\frac{3}{2}\right\}$ (cf. [33]) satisfies (i)-(iii) for any $\mathscr{I} \subset \mathbb{N}$. In certain applications it may be advantageous to choose large domains, and the main restriction on this is the value $N_{\mathscr{I}}=\min \mathscr{I}$. If $\mathscr{I}$ were the set of all prime numbers larger than 100 , say, then the disc $D=\{z \in \mathbb{C}:|z|<100\}$ also satisfies (i)-(iii).

In general, if $f$ is holomorphic on a complex domain $D$ satisfying (i)-(iii), then $\mathscr{L}_{\mathscr{I}, \beta} f$ is holomorphic on some open neighbourhood of $D$. A suitably chosen space of functions holomorphic on $D$, and satisfying some prescribed condition on the boundary of $D$, will then be invariant under $\mathscr{L}_{\mathscr{I}, \beta}$. Ruelle [38] worked with the disc algebra $A_{\infty}(D)$ of holomorphic functions which extend continuously to the boundary of $D$, equipped with the supremum norm. This space has since been the most common choice, although Bergman, Dirichlet, or Hardy spaces (see [12]) are also invariant under $\mathscr{L}_{\mathscr{I}, \beta}$, as are various more exotic (in general nonnormable) spaces (cf. [37, Chapter VIII, § 2, 3]). However, for many reasons it is preferable to work with a Hilbert space. If $D$ is a disc of radius $r$, centred at $c$, and in addition satisfying (i) and (ii) above, then a convenient choice is the Hardy space $H^{2}(D)$ of those functions holomorphic on $D$ and such that

$$
\sup _{\varrho<r} \frac{1}{2 \pi} \int_{0}^{2 \pi}\left|f\left(c+\varrho e^{i t}\right)\right|^{2} d t<\infty .
$$

Note that any function holomorphic on an open neighbourhood of $D$ is automatically in $H^{2}(D)$. The inner product on $H^{2}(D)$ is defined by

$$
(f, g)=\frac{1}{2 \pi} \int_{0}^{2 \pi} f\left(c+r e^{i t}\right) \overline{g\left(c+r e^{i t}\right)} d t,
$$

which is well defined since any element of $H^{2}(D)$ extends as an $L^{2}$ function of $\partial D$.

The very freedom in choosing these holomorphic function spaces means that none of them are canonical. By contrast $C^{\infty}[0,1]$ and $C^{\omega}[0,1]$ are both canonical, although neither of these is normable. Therefore the holomorphic function spaces may usefully be considered as intermediate spaces, on which certain properties of the transfer operator $\mathscr{L}_{\mathscr{I}, \beta}$ are more readily apparent. The intermediate nature of these spaces means that we do not strive for the utmost generality in our choice of the domain $D$. For simplicity we will only consider the Hilbert space $H^{2}(D)$ and the Banach space $A_{\infty}(D)$, where $D$ is a disc satisfying conditions (i) and (ii). When acting on either of these spaces, it will be seen that the operator $\mathscr{L}_{\mathscr{I}, \beta}$ is trace class (and hence compact), a concept we now briefly review.

Given a compact operator $L: H \rightarrow H$ on a Hilbert space $H$, define the $n$th approximation number (or $n$th singular value) to be $s_{n}(L)=\min \{\|L-F\|: F$ is a linear operator of rank at most $n-1\}$. Then $L$ has a norm convergent expansion $L=\sum_{n} s_{n}(L)\left(\varphi_{n}, \cdot\right) \psi_{n}$ for orthonormal sets $\left\{\varphi_{n}\right\},\left\{\psi_{n}\right\}$ (cf. [45, Theorem 1.4]). For $p>0$, the Schatten-von Neumann ideal $\mathscr{S}_{p}(H)$ is the collection of those operators for which $\sum_{n} s_{n}(L)^{p}<\infty$. The case $p=1$ is of particular importance; $L$ is said to be trace class if $\sum_{n} s_{n}(L)<\infty$, in which case its trace $\operatorname{tr}(L)=\sum_{n} s_{n}(L)\left(\varphi_{n}, \psi_{n}\right)$ is well defined. Lidskii's theorem then asserts that $\operatorname{tr}(L)=\sum_{n} \lambda_{n}(L)$, where the $\lambda_{n}(L)$ are the non-zero eigenvalues of $L$, counted 
with multiplicity. The Fredholm determinant for $L$ is an entire function of $z$ defined as $\exp [\operatorname{tr}(\log (I-z L))]$ for small $z$, and then by analytic continuation. Its zeros are precisely the reciprocals of the non-zero eigenvalues of $L$, the order of any zero being the algebraic multiplicity of the corresponding eigenvalue.

Grothendieck $[18,19]$ generalised the notion of trace class operators to Banach spaces $B$. If $L: B \rightarrow B$ can be written as $L(f)=\sum_{n} l_{n}(f) f_{n}$, for some $l_{n} \in B^{\prime}$ and $f_{n} \in B$ satisfying $\sum_{n}\left\|l_{n}\right\|_{B^{\prime}}\left\|f_{n}\right\|_{B}<\infty$, then $L$ is said to be nuclear. If $\sum_{n}\left(\left\|l_{n}\right\|_{B^{\prime}}\left\|f_{n}\right\|_{B}\right)^{p}<\infty$, then $L$ is called nuclear of order $p$. If $L$ is nuclear of order $p$ for every $p>0$ then it is said to be nuclear of order 0 . The class of nuclear operators of order $\frac{2}{3}$ (the Grothendieck trace class) is of particular importance, for in that case the trace $\operatorname{tr}(L)=\sum_{n} l_{n}\left(f_{n}\right)$ is well defined, and independent of the sequences $l_{n}$ and $f_{n}$. Moreover, for such operators Grothendieck [18] proved that the analogue of Lidskii's theorem holds, namely $\operatorname{tr}(L)=\sum_{n} \lambda_{n}(L)$. The Fredholm determinant can also be defined for such operators $L$, in the same way as for trace class operators on Hilbert space, and its zeros are again related to the eigenvalues of $L$ as above.

If $\left\|l_{n}\right\|_{B^{\prime}}\left\|f_{n}\right\|_{B}=O\left(\gamma^{n}\right)$ for some $0<\gamma<1$, we will say that $L$ is exponentially nuclear (such operators are clearly nuclear of order 0 ). Moreover we have the tail estimate $\sum_{n=N}^{\infty}\left\|l_{n}\right\|_{B^{\prime}}\left\|f_{n}\right\|_{B}=O\left(\gamma^{N}\right)$, so that $s_{N}(L)=O\left(\gamma^{N}\right)$, and hence $\sum_{n} s_{n}(L)<\infty$. Therefore an exponentially nuclear operator on a Hilbert space is of trace class.

Proposition 1. Suppose $\mathscr{I} \subset \mathbb{N}$ is non-empty, and $\operatorname{Re}(\beta)>\theta_{\mathscr{I}}$. Let $D \subset \mathbb{C}$ be a disc satisfying conditions (i) and (ii).

(a) $\mathscr{L}_{\mathscr{I}, \beta}: H^{2}(D) \rightarrow H^{2}(D)$ belongs to every Schatten-von Neumann ideal $\mathscr{S}_{p}\left(H^{2}(D)\right)$, with $p>0$, and in particular is trace class.

(b) $\mathscr{L}_{\mathscr{J}, \beta}: A_{\infty}(D) \rightarrow A_{\infty}(D)$ is nuclear of order 0 , and in particular is a Grothendieck trace class operator.

Proof. The assumptions on $D$ guarantee that if $f$ is holomorphic on $D$ then $\mathscr{L}_{\mathscr{I}, \beta} f$ is holomorphic on an open neighbourhood of $D$. In particular we may choose an open disc $D^{\prime}$, concentric with $D$, and with strictly larger radius, such that $\mathscr{L}_{\mathscr{I}, \beta} f$ is holomorphic on $D^{\prime}$. Let $r$ and $r^{\prime}$ denote the radii of $D$ and $D^{\prime}$ respectively, and let $c$ denote their common centre. We have the Taylor series

$$
\mathscr{L}_{\mathscr{I}, \beta} f(z)=\sum_{k=0}^{\infty} \psi_{k}(f) g_{k}(z),
$$

where

$$
\psi_{k}(f)=\frac{\left(\mathscr{L}_{\mathscr{I}, \beta} f\right)^{(k)}(c)}{k !}, \quad g_{k}(z)=(z-c)^{k} .
$$

Now $\psi_{k}(f)=O\left(\left(r^{\prime}\right)^{-k}\right)$, since $\mathscr{L}_{\mathscr{I}, \beta} f$ is holomorphic on $D^{\prime}$.

In particular, if $E$ denotes either $H^{2}(D)$ or $A_{\infty}(D)$ then $\mathscr{L}_{\mathscr{I}, \beta} f \in E$, and moreover $\left\|\psi_{k}\right\|_{E^{\prime}}=O\left(\left(r^{\prime}\right)^{-k}\right)$. Clearly also $g_{k} \in E$, and $\left\|g_{k}\right\|_{E}=r^{k}$. Therefore $\left\|g_{k}\right\|_{E}\left\|\psi_{k}\right\|_{E^{\prime}}=O\left(\left(r / r^{\prime}\right)^{k}\right)$, so that $\mathscr{L}_{\mathscr{I}, \beta}$ is exponentially nuclear. The discussion preceding this proposition then implies that $\mathscr{L}_{\mathscr{I}, \beta}$ is (Grothendieck) trace class.

We now consider the action of $\mathscr{L}_{\mathscr{I}, \beta}$ on the spaces $C^{\infty}[0,1]$ and $C^{\omega}[0,1]$. These are not Banach spaces, so care is needed in the definition of its spectrum, 
which we will always understand in the sense of Allan [1] (see also § A.1 of [4]). The spectrum of a linear operator $L: E \rightarrow E$ on a topological vector space $E$ will be denoted by $\sigma(L \mid E)$.

Proposition 2. Suppose $\mathscr{I} \subset \mathbb{N}$ is non-empty, and $\operatorname{Re}(\beta)>\theta_{\mathscr{I}}$. Both

$$
\mathscr{L}_{\mathscr{Y}, \beta}: C^{\infty}[0,1] \rightarrow C^{\infty}[0,1] \quad \text { and } \quad \mathscr{L}_{\mathscr{I}, \beta}: C^{\omega}[0,1] \rightarrow C^{\omega}[0,1]
$$

have countable spectrum, the only accumulation point being at 0 .

Proof. First we consider the operator $\mathscr{L}_{\mathscr{Y}, \beta}$ acting on the space $C^{\omega}[0,1]$. Let $\Delta_{j}$ be a decreasing sequence of open rectangles with $\bigcap_{j} \Delta_{j}=[0,1]$, such that $\overline{\Delta_{j}} \subset \operatorname{int}\left(\bigcap_{n \in \mathscr{I}} S_{n}(\Delta)\right)$ and $-\mathscr{I} \cap \Delta_{j}=\emptyset$. For definiteness we could choose $\Delta_{j}$ to be centred at $a_{j}=\frac{1}{2}+1 / j$, of height $1 / j$ and length $1+\frac{1}{2} \sqrt{\left(2 a_{j}+3\right)\left(2 a_{j}-1\right)}$.

By Riemann's mapping theorem, there is a holomorphic diffeomorphism $\Phi_{j}: \operatorname{int}\left(\bigcap_{n \in \mathscr{I}} S_{n}\left(\Delta_{j}\right)\right) \rightarrow \mathbb{D}(0,1)$ sending the simply connected Jordan domain $\operatorname{int}\left(\bigcap_{n \in \mathscr{I}} S_{n}\left(\Delta_{j}\right)\right)$ onto the open unit disc $\mathbb{D}(0,1)$. There is some $0<\varrho_{j}<1$ such that $\Phi\left(\Delta_{j}\right) \subset \mathbb{D}\left(0, \varrho_{j}\right)$, the open disc of radius $\varrho_{j}$ centred at 0 . Let $C_{j}$ denote the composition operator $C_{j} f=f \circ \Phi_{j}$. The Riemann mapping $\Phi_{j}$ extends to a boundary homeomorphism $\partial\left(\bigcap_{n \in \mathscr{I}} S_{n}\left(\Delta_{j}\right)\right) \rightarrow S^{1}$ (see [23, Theorem 17.5.3]). This ensures that $C_{j}$ is a continuous operator $A_{\infty}(\mathbb{D}(0,1)) \rightarrow A_{\infty}\left(\operatorname{int}\left(\bigcap_{n \in \mathscr{I}} S_{n}\left(\Delta_{j}\right)\right)\right)$, with unit norm. The operator $C_{j}^{-1}$ is also continuous, for the same reason.

By an argument analogous to that of Proposition 1 we see that $C_{j} \circ \mathscr{L}_{\mathscr{I}, \beta} \circ C_{j}^{-1}$ is exponentially nuclear when acting on the space $A_{\infty}\left(\Phi\left(\Delta_{j}\right)\right)$. From the continuity of $C_{j}$ and $C_{j}^{-1}$ we deduce that $\mathscr{L}_{\mathscr{I}, \beta}: A_{\infty}\left(\Delta_{j}\right) \rightarrow A_{\infty}\left(\Delta_{j}\right)$ is also exponentially nuclear. It follows that the non-zero eigenvalues of $\mathscr{L}_{\mathscr{F}, \beta}: A_{\infty}\left(\Delta_{j}\right) \rightarrow A_{\infty}\left(\Delta_{j}\right)$, counted with algebraic multiplicity, are precisely the reciprocals of the zeros, again counted with multiplicity, of the Fredholm determinant $D_{\mathscr{I}, \beta}(z)$ of $\mathscr{L}_{\mathscr{I}, \beta}$. But by a result of Ruelle [38], the Fredholm determinant for a transfer operator can be expressed in terms of the periodic points of the underlying dynamical system as

$$
D_{\mathscr{I}, \beta}(z)=\exp -\sum_{n=1}^{\infty} \frac{z^{n}}{n} \sum_{\substack{T^{n} x=x \\ x \in \Lambda_{\mathscr{J}}}} \frac{\prod_{i=0}^{n-1}\left(T^{i} x\right)^{2 \beta}}{1-(-1)^{n} \prod_{i=0}^{n-1}\left(T^{i} x\right)^{2}},
$$

an expression which is manifestly independent of the particular domain $\Delta_{j}$. Thus the spectrum of $\mathscr{L}_{\mathscr{I}, \beta}: A_{\infty}\left(\Delta_{j}\right) \rightarrow A_{\infty}\left(\Delta_{j}\right)$ is independent of $\Delta_{j}$. Let $\left\{\lambda_{i}\right\}_{i \in \mathbb{N}} \cup\{0\}$ denote this common spectrum.

Now $C^{\omega}[0,1]$ is topologised as the inductive limit of the injective inductive system of Banach spaces $E_{j}:=A_{\infty}\left(\Delta_{j}\right)$. Each $\mathscr{L}_{\mathscr{I}, \beta}: E_{j} \rightarrow E_{j}$ is nuclear, and hence compact, and these operators all have identical spectrum $\left\{\lambda_{i}\right\}_{i \in \mathbb{N}} \cup\{0\}$. A result of Bandtlow [4, Corollary A.7.11] then allows us to deduce that the spectrum of $\mathscr{L}_{\mathscr{I}, \beta}: C^{\omega}[0,1] \rightarrow C^{\omega}[0,1]$ is also $\left\{\lambda_{i}\right\}_{i \in \mathbb{N}} \cup\{0\}$.

To treat the operator $\mathscr{L}_{\mathscr{S}, \beta}: C^{\infty}[0,1] \rightarrow C^{\infty}[0,1]$, we first note that $C^{\infty}[0,1]$ is the projective limit of the countable injective projective system of Banach spaces $C^{k}[0,1]$. This means, by [4, Proposition A.7.20], that the spectrum $\sigma\left(\mathscr{L}_{\mathscr{I}, \beta} \mid C^{\infty}[0,1]\right)$ is the intersection of the spectra $\sigma\left(\mathscr{L}_{\mathscr{I}, \beta} \mid C^{k}[0,1]\right)$, with $k \in \mathbb{N}$.

We claim that $\mathscr{L}_{\mathscr{I}, \beta}: C^{k}[0,1] \rightarrow C^{k}[0,1]$ is quasicompact, and that its essential spectral radius shrinks to 0 as $k \rightarrow \infty$. This will essentially follow from another result of Ruelle [42]. Since $\operatorname{Re}(\beta)>\theta_{\mathscr{I}}$ then condition (i) on p.176 of [42] is 
satisfied for the operator $\mathscr{L}_{\mathscr{I}, \beta}$, and also for $\mathscr{L}_{\mathscr{I}, \beta}^{2}$. Now $\mathscr{L}_{\mathscr{I}, \beta}^{2}$ is a transfer operator for the infinite iterated function system determined by the collection of strict contractions

$$
T_{m, n}(z)=\left(m+\frac{1}{z+n}\right)^{-1} \text { for }(m, n) \in \mathscr{I}^{2} .
$$

Since $\left|T_{m, n}^{\prime}(z)\right| \leqslant \frac{1}{4}$ for all $(m, n) \in \mathscr{I}^{2}$ and $z \in[0,1]$, the results of [42] can be applied to $\mathscr{L}_{\mathscr{I}, \beta}^{2}$ (the subtlety here is that if $1 \in \mathscr{I}$ then Ruelle's results would not apply verbatim to $\mathscr{L}_{\mathscr{I}, \beta}$, since $T_{1}(z)=1 /(z+1)$ is not a strict contraction). By Theorem 1.3 of [42] we see that $\mathscr{L}_{\mathscr{I}, \beta}^{2}: C^{k}[0,1] \rightarrow C^{k}[0,1]$ is quasicompact, with essential spectral radius bounded by $c\left(\frac{1}{4}\right)^{k}$, where $c>0$ is independent of $k$. If we consider both $\mathscr{L}_{\mathscr{I}, \beta}$ and $\mathscr{L}_{\mathscr{J}, \beta}^{2}$ as acting on the Calkin algebra (that is, bounded operators modulo compact ones), then an application of the spectral mapping theorem shows that $\mathscr{L}_{\mathscr{I}, \beta}: C^{k}[0,1] \rightarrow C^{k}[0,1]$ is also quasicompact, with essential spectral radius bounded by $\sqrt{c}\left(\frac{1}{2}\right)^{k}$.

In particular, the essential spectral radius of $\mathscr{L}_{\mathscr{I}, \beta}$ does tend to 0 as $k \rightarrow \infty$, so that $\sigma\left(\mathscr{L}_{\mathscr{I}, \beta} \mid C^{\infty}[0,1]\right)=\bigcap_{k=1}^{\infty} \sigma\left(\mathscr{L}_{\mathscr{I}, \beta} \mid C^{k}[0,1]\right)$ is countable, its only accumulation point being at 0 .

REMARK 1. In the course of the above proof it was shown that the spectrum of $\mathscr{L}_{\mathscr{I}, \beta}$ on $C^{\omega}[0,1]$ coincides with that on $A_{\infty}(D)$, for suitable domains $D$. In fact it can be proved that the spectrum of $\mathscr{L}_{\mathscr{J}, \beta}$ on each of the spaces $C^{\infty}[0,1], C^{\omega}[0,1]$, $H^{2}(D)$ and $A_{\infty}(D)$ is identical. The proof of this is postponed until Theorem 3, when the Hilbert space $\mathscr{H}_{\mathscr{I}, \beta}$ will also intervene.

\section{The integral operator and integral transform}

The following lemma is elementary, and its proof is left as an exercise.

Lemma 1 (Properties of the theta function $\vartheta_{\mathscr{I}}$ ). Suppose $\mathscr{I} \subset \mathbb{N}$ is non-empty. Let $\vartheta_{\mathscr{I}}(t)=\sum_{n \in \mathscr{I}} e^{-n t}$ be the corresponding theta function. Then

(a) $\vartheta_{\mathscr{I}}: \mathbb{R}^{+} \rightarrow \mathbb{R}^{+}$is monotone decreasing, with $\lim _{t \rightarrow \infty} \vartheta_{\mathscr{I}}(t)=0$,

(b) $\lim _{t \backslash 0} \vartheta_{\mathscr{I}}(t)=\infty$ if and only if $\mathscr{I}$ is infinite,

(c) $e^{-N_{\mathscr{f}} t} \leqslant \vartheta_{\mathscr{I}}(t) \leqslant\left(1-e^{-1}\right)^{-1} e^{-N_{\mathscr{f}} t}$ for all $t \geqslant 1$.

The following lemma is a standard tool in Mellin transform theory, and will be used several times. Its proof follows from the simple change of variable $u=a t$.

Lemma 2 (Mellin transform trick). For $\operatorname{Re}(s)>0, a>0$, and any locally integrable function $g$ for which either side of the equality is finite,

$$
\int_{0}^{\infty} t^{s-1} g(a t) d t=\frac{1}{a^{s}} \int_{0}^{\infty} u^{s-1} g(u) d u .
$$

An immediate application is to the following.

Lemma 3. Suppose $\mathscr{I} \subset \mathbb{N}$ is non-empty. If $\operatorname{Re}(s)>\max \left(0,2 \theta_{\mathscr{I}}\right)$ then

$$
\zeta_{\mathscr{I}}(s, z)=\frac{1}{\Gamma(s)} \int_{0}^{\infty} t^{s-1} e^{-z t} d m_{\mathscr{I}}(t) .
$$


Proof. Since $\operatorname{Re}(s)>0$, we have

$$
\zeta_{\mathscr{I}}(s, z) \Gamma(s)=\sum_{n \in \mathscr{I}} \frac{1}{(z+n)^{s}} \int_{0}^{\infty} u^{s-1} e^{-u} d u .
$$

Applying Lemma 2 and then interchanging integration and summation (which is justified since both are absolutely convergent) gives

$$
\begin{aligned}
\zeta_{\mathscr{I}}(s, z) \Gamma(s) & =\sum_{n \in \mathscr{I}} \int_{0}^{\infty} t^{s-1} e^{-t(z+n)} d t \\
& =\int_{o}^{\infty} t^{s-1} e^{-z t} \sum_{n \in \mathscr{I}} e^{-t n} d t \\
& =\int_{0}^{\infty} t^{s-1} e^{-z t} d m_{\mathscr{I}}(t) .
\end{aligned}
$$

REMARK 2. The case $z=0$ in Lemma 3 yields the following integral expression for the zeta function $\zeta_{\mathscr{I}}(s)$ :

$$
\zeta_{\mathscr{I}}(s)=\frac{1}{\Gamma(s)} \int_{0}^{\infty} t^{s-1} d m_{\mathscr{I}}(t)
$$

valid for $\operatorname{Re}(s)>\max \left(0,2 \theta_{\mathscr{I}}\right)$.

Lemma 4. Suppose $\mathscr{I} \subset \mathbb{N}$ is non-empty, and $\operatorname{Re}(\beta)>\max \left(0, \theta_{\mathscr{I}}\right)$. If $\varphi \in L^{2}\left(m_{\mathscr{I}}\right)$ then $\mathscr{T}_{\mathscr{I}, \beta}(\varphi)$ is holomorphic in the right half-plane $R_{\mathscr{I}}=\left\{z \in \mathbb{C}: \operatorname{Re}(z)>-\frac{1}{2} N_{\mathscr{I}}\right\}$.

Proof. Suppose $\varphi \in L^{2}\left(m_{\mathscr{I}}\right)$. By Lemma 1.1 of [25], it suffices to show that

$$
\mathscr{T}_{\mathscr{I}, \beta} \varphi(z)=\int_{0}^{\infty} s^{\beta-1 / 2} e^{-s z} \varphi(s) d m_{\mathscr{I}}(s)=\int_{0}^{\infty} s^{\beta-1 / 2} e^{-s z} \varphi(s) \vartheta_{\mathscr{A}}(s) d s
$$

is uniformly convergent in $z$, on any compact subset of $R_{\mathscr{I}}$. To do this we will split the range of integration, considering separately the integrals $\int_{1}^{\infty}$ and $\int_{0}^{1}$.

We will first show that $\int_{1}^{\infty}\left|s^{\beta-1 / 2} e^{-s z} \varphi(s)\right| \vartheta_{\mathscr{I}}(s) d s<\infty$, uniformly on any compact subset of $R_{\mathscr{I}}$.

Since $\varphi \in L^{2}\left(m_{\mathscr{I}}\right)$ then $\int_{0}^{\infty}|\varphi(s)|^{2} \vartheta_{\mathscr{I}}(s) d s<\infty$, and so $|\varphi(s)|^{2} \vartheta_{\mathscr{I}}(s) \rightarrow 0$ as $s \rightarrow \infty$. By Lemma 1(c) we deduce that $|\varphi(s)| \leqslant e^{s N_{\mathscr{S}} / 2}$ for all sufficiently large $s$. Consequently, there exists $K>0$ such that

$$
\int_{1}^{\infty}\left|s^{\beta-1 / 2} e^{-s z} \varphi(s)\right| \vartheta_{\mathscr{I}}(s) d s \leqslant K \int_{1}^{\infty} s^{\beta-1 / 2} e^{-s\left(\operatorname{Re}(z)+N_{\mathscr{J}} / 2\right)} d s,
$$

and this integral is convergent for $\operatorname{Re}(z)>-\frac{1}{2} N_{\mathscr{I}}$. The convergence is uniform on any compact subset of $R_{\mathscr{I}}$ (indeed on any half-plane $\operatorname{Re}(z) \geqslant-\frac{1}{2} N_{\mathscr{I}}+\delta$, for $\delta>0$ ).

We now consider the integral $\int_{0}^{1}\left|s^{\beta-1 / 2} e^{-s z} \varphi(s)\right| d m_{\mathscr{I}}(s)$. We first claim that $s \mapsto \varphi(s) s^{\beta-1 / 2}$ is in $L^{1}\left(m_{\mathscr{I}}\right)$. To prove this it suffices to show, by the CauchySchwarz inequality, that $s^{\beta-1 / 2} \in L^{2}\left(m_{\mathscr{I}}\right)$. An application of Remark 2 gives

$$
\int_{0}^{\infty}\left|s^{\beta-1 / 2}\right|^{2} d m_{\mathscr{I}}(s)=\int_{0}^{\infty} s^{2 \operatorname{Re}(\beta)-1} d m_{\mathscr{I}}(s)=\zeta_{\mathscr{I}}(2 \operatorname{Re}(\beta)) \Gamma(2 \operatorname{Re}(\beta))<\infty
$$

for $\operatorname{Re}(\beta)>\max \left(0, \theta_{\mathscr{I}}\right)$, as required. 
For $\operatorname{Re}(z)>-\frac{1}{2} N_{\mathscr{I}}$ we have

$$
\begin{aligned}
\int_{0}^{1}\left|s^{\beta-1 / 2} e^{-s z} \varphi(s)\right| d m_{\mathscr{I}}(s) & \leqslant e^{N_{\mathscr{I}} / 2} \int_{0}^{1} s^{\operatorname{Re}(\beta)-1 / 2}|\varphi(s)| d m_{\mathscr{I}}(s) \\
& \leqslant e^{N_{\mathscr{I}} / 2} \int_{0}^{\infty} s^{\operatorname{Re}(\beta)-1 / 2}|\varphi(s)| d m_{\mathscr{I}}(s) \\
& <\infty
\end{aligned}
$$

since $\varphi(s) s^{\beta-1 / 2} \in L^{1}\left(m_{\mathscr{I}}\right)$. Indeed the convergence of this integral is uniform on all of $R_{\mathscr{I}}$.

We have checked that the integral $\int_{0}^{\infty} s^{\beta-1 / 2} e^{-s z} \varphi(s) d m_{\mathscr{I}}(s)$ is absolutely convergent, uniformly on any compact subset of $R_{\mathscr{I}}$, so we have the required result.

The integral operator $\mathscr{K}_{\mathscr{Y}, \beta}$ will always act on the space $L^{2}\left(m_{\mathscr{I}}\right)$, which we now show it leaves invariant.

Lemma 5. Suppose $\mathscr{I} \subset \mathbb{N}$ is non-empty, and $\operatorname{Re}(\beta)>\max \left(0, \theta_{\mathscr{I}}\right)$. The integral operator $\mathscr{K}_{\mathscr{I}, \beta}$

(a) preserves the space $L^{2}\left(m_{\mathscr{I}}\right)$,

(b) is trace class when acting on $L^{2}\left(m_{\mathscr{I}}\right)$,

(c) is selfadjoint in the case where $\beta$ is real.

Proof. Part (a) will follow from part (b), which we now prove.

The straightforward expansion

$$
\mathscr{K}_{\mathscr{I}, \beta} \varphi(t)=\sum_{k=0}^{\infty} \frac{(-1)^{k} t^{k+\beta-1 / 2}}{k ! \Gamma(k+2 \beta)} \int_{0}^{\infty} s^{k+\beta-1 / 2} \varphi(s) d m_{\mathscr{I}}(s)
$$

is in fact not a nuclear representation. However, the Bessel function can be expanded (cf. [47, p. 8]) in terms of Laguerre polynomials $L_{n}^{(2 \beta-1)}$ (which form an orthogonal basis for $\left.L^{2}\left(\mathbb{R}^{+}, d s\right)\right)$ as

$$
G_{\beta}(s, t)=\mathscr{J}_{2 \beta-1}(2 \sqrt{s t})=(s t)^{\beta-1 / 2} e^{-t} \sum_{n=0}^{\infty} \frac{L_{n}^{(2 \beta-1)}(s) t^{n}}{\Gamma(n+2 \beta)} .
$$

This gives the representation

$$
\mathscr{K}_{\mathscr{Y}, \beta} \varphi=\sum_{n=0}^{\infty} l_{n}(\varphi) f_{n}
$$

where

$$
f_{n}(s)=s^{\beta-1 / 2} L_{n}^{(2 \beta-1)}(s), \quad l_{n}(\varphi)=\int_{0}^{\infty} \frac{t^{n+\beta-1 / 2} e^{-t}}{\Gamma(n+2 \beta)} \varphi(t) d m_{\mathscr{I}}(t) .
$$

A calculation (cf. [34, p. 155]), again using Lemma 2, reveals this to be an exponentially nuclear representation, with the exponential decay of the $\left\|l_{n}\right\|$ dominating the exponential growth of the $\left\|f_{n}\right\|$. The discussion preceding Proposition 1 then implies that $\mathscr{L}_{\mathscr{I}, \beta}$ is trace class.

Part (c) is clear since $G_{\beta}(s, t)=\overline{G_{\beta}(t, s)}$ for $\beta \in \mathbb{R}$. 
5. The transfer operator on the space of integral transforms

Theorem 1. Suppose $\mathscr{I} \subset \mathbb{N}$ is non-empty, and $\operatorname{Re}(\beta)>\max \left(0, \theta_{\mathscr{I}}\right)$. Then

(a) the transfer operator $\mathscr{L}_{\mathscr{I}, \beta}$ preserves the space $\mathscr{H}_{\mathscr{I}, \beta}=\mathscr{T}_{\mathscr{I}, \beta}\left(L^{2}\left(m_{\mathscr{I}}\right)\right)$,

(b) if $\mathscr{K}_{\mathscr{I}, \beta}: L^{2}\left(m_{\mathscr{I}}\right) \rightarrow L^{2}\left(m_{\mathscr{I}}\right)$ is the integral operator with kernel function $G_{\beta}$, then

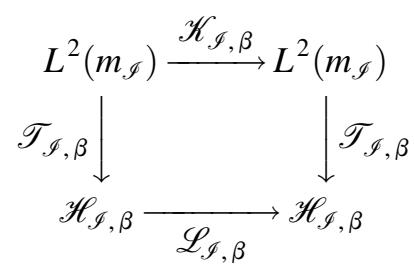

is a commutative diagram.

Proof. At several points during this proof we swap order of summation, or interchange summation and integration. On each occasion this is justified by absolute convergence, stemming from the hypothesis $\operatorname{Re}(\beta)>\max \left(0, \theta_{\mathscr{I}}\right)$. Now

$$
\begin{aligned}
\mathscr{L}_{\mathscr{I}, \beta} \circ \mathscr{T}_{\mathscr{I}, \beta} \varphi(z) & =\sum_{n \in \mathscr{I}}\left(\frac{1}{z+n}\right)^{2 \beta} \mathscr{T}_{\mathscr{I}, \beta} \varphi\left(\frac{1}{z+n}\right) \\
& =\sum_{n \in \mathscr{I}}\left(\frac{1}{z+n}\right)^{2 \beta} \int_{0}^{\infty} s^{\beta-1 / 2} e^{-s /(z+n)} \varphi(s) d m_{\mathscr{I}}(s) \\
& =\int_{0}^{\infty} s^{\beta-1 / 2} \varphi(s) \sum_{n \in \mathscr{I}}\left(\frac{1}{z+n}\right)^{2 \beta} e^{-s /(z+n)} d m_{\mathscr{I}}(s) .
\end{aligned}
$$

Manipulating part of the integrand we have

$$
\begin{aligned}
\sum_{n \in \mathscr{I}}\left(\frac{1}{z+n}\right)^{2 \beta} e^{-s /(z+n)} & =\sum_{n \in \mathscr{I}}\left(\frac{1}{z+n}\right)^{2 \beta} \sum_{k=0}^{\infty}\left(\frac{-s}{z+n}\right)^{k} \frac{1}{k !} \\
& =\sum_{k=0}^{\infty} \sum_{n \in \mathscr{I}}\left(\frac{1}{z+n}\right)^{k+2 \beta} \frac{(-s)^{k}}{k !} \\
& =\sum_{k=0}^{\infty} \frac{(-s)^{k}}{k !} \zeta_{\mathscr{I}}(k+2 \beta, z) \\
& =\sum_{k=0}^{\infty} \frac{(-s)^{k}}{k ! \Gamma(k+2 \beta)} \int_{0}^{\infty} t^{k+2 \beta-1} e^{-z t} d m_{\mathscr{I}}(t),
\end{aligned}
$$

where Lemma 3 was used for the final step. 
Substituting into (6) gives us

$$
\begin{aligned}
\mathscr{L}_{\mathscr{I}, \beta} \circ \mathscr{T}_{\mathscr{I}, \beta} \varphi(z) & =\int_{s=0}^{\infty} \int_{t=0}^{\infty} s^{\beta-1 / 2} \varphi(s) \sum_{k=0}^{\infty} \frac{(-s)^{k}}{k ! \Gamma(k+2 \beta)} t^{k+2 \beta-1} e^{-z t} d m_{\mathscr{I}}(t) d m_{\mathscr{I}}(s) \\
= & \int_{s=0}^{\infty} \int_{t=0}^{\infty} \varphi(s) G_{\beta}(s, t) t^{\beta-1 / 2} e^{-z t} d m_{\mathscr{I}}(t) d m_{\mathscr{I}}(s) \\
= & \int_{t=0}^{\infty} t^{\beta-1 / 2} e^{-z t}\left[\int_{s=0}^{\infty} G_{\beta}(s, t) \varphi(s) d m_{\mathscr{I}}(s)\right] d m_{\mathscr{I}}(t) \\
& =\mathscr{T}_{\mathscr{I}, \beta} \circ \mathscr{K}_{\mathscr{I}, \beta} \varphi(z) .
\end{aligned}
$$

So indeed the operators $\mathscr{L}_{\mathscr{I}, \beta}$ and $\mathscr{K}_{\mathscr{I}, \beta}$ are conjugated by the integral transform $\mathscr{T}_{\mathscr{I}, \beta}$, and in particular $\mathscr{L}_{\mathscr{I}, \beta}$ preserves the space $\mathscr{H}_{\mathscr{I}, \beta}$.

REMARK 3. It is now clear why the exponent $\beta-\frac{1}{2}$ was chosen in the integral transform $\mathscr{T}_{\mathscr{I}, \beta} \varphi(z)=\int_{0}^{\infty} s^{\beta-1 / 2} e^{-s z} \varphi(s) d m_{\mathscr{I}}(s)$. It ensures that $\mathscr{L}_{\mathscr{I}, \beta}$ is conjugate to an integral operator with symmetric kernel.

THeOREM 2. Let $\mathscr{I}$ be a non-empty subset of $\mathbb{N}$. Suppose $\beta \in \mathbb{R}$ with $\beta>\max \left(0, \theta_{\mathscr{I}}\right)$. Then the operator $\mathscr{L}_{\mathscr{I}, \beta}: \mathscr{H}_{\mathscr{I}, \beta} \rightarrow \mathscr{H}_{\mathscr{I}, \beta}$ is conjugate to a selfadjoint operator, and in particular has real spectrum.

This spectrum is a sequence of real numbers union its accumulation point at 0.

Proof. The continuous conjugacy of Theorem 1 between the integral operator $\mathscr{K}_{\mathscr{I}, \beta}$ and the transfer operator $\mathscr{L}_{\mathscr{I}, \beta}: \mathscr{H}_{\mathscr{I}, \beta} \rightarrow \mathscr{H}_{\mathscr{I}, \beta}$ implies that their spectra coincide.

Now $\mathscr{K}_{\mathscr{I}, \beta}$ is trace class, by Lemma $5(\mathrm{~b})$, so in particular it is compact. Therefore the only non-discrete point in the spectrum of $\mathscr{K}_{\mathscr{I}, \beta}$ is the accumulation point at 0 . Also, $\mathscr{K}_{\mathscr{I}, \beta}$ is selfadjoint, by Lemma 5(c), so its spectrum is real.

\section{The full $C^{\infty}$ spectrum of the transfer operator}

In this section it will be shown that the space $\mathscr{H}_{\mathscr{I}, \beta}$ is large enough to capture the full spectrum of $\mathscr{L}_{\mathscr{I}, \beta}$ acting on $C^{\infty}[0,1]$ (and on various spaces of analytic functions), as well as the discrete part of the spectrum of $\mathscr{L}_{\mathscr{I}, \beta}$ acting on either $C^{k}[0,1]$ or $C^{\alpha}[0,1]$. This will follow from the fact that $\mathscr{H}_{\mathscr{Y}, \beta}$ is densely and continuously embedded in these various spaces, a result we now prove.

Lemma 6. Let $\mathscr{I} \subset \mathbb{N}$ be non-empty, and $\operatorname{Re}(\beta)>\max \left(0, \theta_{\mathscr{I}}\right)$. Suppose the open disc $D$ satisfies $[0,1] \subset D$ and $\bar{D} \subset R_{\mathscr{F}}$. Let $E$ denote any of the spaces $A_{\infty}(D), \quad H^{2}(D), \quad C^{\omega}[0,1], \quad C^{\infty}[0,1], \quad C^{k}[0,1] \quad($ for $k \in \mathbb{N}), \quad$ or $C^{\alpha}[0,1]$ (for $0<\alpha \leqslant 1)$. The natural inclusion $\iota_{E}: \mathscr{H}_{\mathscr{I}, \beta} \rightarrow E$ is continuous, and $\iota_{E}\left(\mathscr{H}_{\mathscr{I}, \beta}\right)$ is a dense subspace of $E$.

Proof. First we show that the inclusions $\iota_{E}$ are continuous. For any $k \in \mathbb{N}$ and $\alpha \in(0,1]$, each inclusion in the chain

$$
A_{\infty}(D) \hookrightarrow H^{2}(D) \hookrightarrow C^{\omega}[0,1] \hookrightarrow C^{\infty}[0,1] \hookrightarrow C^{k}[0,1] \hookrightarrow C^{\alpha}[0,1]
$$


is continuous, as is readily verified, so it suffices to check the continuity of $\iota_{A_{\infty}(D)}: \mathscr{H}_{\mathscr{I}, \beta} \rightarrow A_{\infty}(D)$. In the definition of $\mathscr{H}_{\mathscr{I}, \beta}$ we declared $\mathscr{T}_{\mathscr{I}, \beta}$ to be a Hilbert space isomorphism; so $\left\|\mathscr{T}_{\mathscr{I}, \beta} \varphi\right\|_{\mathscr{I}, \beta}=\|\varphi\|_{L^{2}\left(m_{\mathscr{f}}\right)}$ for every $\varphi \in L^{2}\left(m_{\mathscr{I}}\right)$, where $\|\cdot\|_{\mathscr{I}, \beta}$ denotes the norm in $\mathscr{H}_{\mathscr{I}, \beta}$.

Now for any $z \in \bar{D}$ and $\operatorname{Re}(\beta)>\max \left(0, \theta_{\mathscr{I}}\right)$, the function $r_{z, \beta}: s \mapsto s^{\beta-1 / 2} e^{-s z}$ belongs to $L^{2}\left(m_{\mathscr{I}}\right)$, since

$$
\begin{aligned}
\int_{0}^{\infty}\left|s^{\beta-1 / 2} e^{-s z}\right|^{2} d m_{\mathscr{I}}(s) & =\int_{0}^{\infty} s^{2 \operatorname{Re}(\beta)-1} e^{-2 s z} \sum_{n \in \mathscr{I}} e^{-n s} d s \\
& =\zeta_{\mathscr{I}}(2 \operatorname{Re}(\beta), 2 z) \Gamma(2 \operatorname{Re}(\beta)),
\end{aligned}
$$

which is finite because $\operatorname{Re}(2 z)>-N_{\mathscr{I}}$, and $\operatorname{Re}(\beta)>\max \left(0, \theta_{\mathscr{I}}\right)$. Indeed there is a constant $C_{D, \beta}>0$ such that $\left\|r_{z, \beta}\right\|_{L^{2}\left(m_{\mathscr{J}}\right)} \leqslant C_{D, \beta}$ for all $z \in \bar{D}$, since $D$ is compactly contained in $R_{\mathscr{I}}$.

For all $\varphi \in L^{2}\left(m_{\mathscr{I}}\right)$ and $z \in \bar{D}$, the Cauchy-Schwarz inequality then gives

$$
\begin{aligned}
\left|\mathscr{T}_{\mathscr{I}, \beta} \varphi(z)\right| & =\left|\int_{0}^{\infty} s^{\beta-1 / 2} e^{-s z} \varphi(s) d m_{\mathscr{I}}(s)\right| \\
& \leqslant\left\|r_{z, \beta}\right\|_{L^{2}\left(m_{\mathscr{P}}\right)}\|\varphi\|_{L^{2}\left(m_{\mathscr{P}}\right)} \\
& \leqslant C_{D, \beta}\left\|\mathscr{T}_{\mathscr{I}, \beta} \varphi\right\|_{\mathscr{I}, \beta} .
\end{aligned}
$$

That is, $\left\|\mathscr{T}_{\mathscr{I}, \beta} \varphi\right\|_{A_{\infty}(D)} \leqslant C_{D, \beta}\left\|\mathscr{T}_{\mathscr{I}, \beta} \varphi\right\|_{\mathscr{I}, \beta}$, so the inclusion $\mathscr{H}_{\mathscr{I}, \beta} \hookrightarrow A_{\infty}(D)$ is continuous, as required.

It now remains to prove that each $\iota_{E}\left(\mathscr{H}_{\mathscr{I}, \beta}\right)$ is dense in $E$. Define $f_{j}(z)=1 /(z+j)$, for $j \in \mathbb{N}$ with $j>N_{\mathscr{I}}$. Each $f_{j} \in E$, and we claim that the linear span of $\left\{f_{j}\right\}_{j>N_{\mathscr{S}}}$ is a dense subspace of $E$. To see this it suffices to show that any monomial $z^{m}$ can be approximated by linear combinations of the $f_{j}$, since the space of all polynomials is certainly dense in $E$. If

$$
g_{p, m}(z)=\frac{\left(p+N_{\mathscr{I}}\right)\left(p+1+N_{\mathscr{I}}\right) \ldots\left(p+m+N_{\mathscr{I}}\right)}{\left(z+p+N_{\mathscr{I}}\right)\left(z+p+1+N_{\mathscr{I}}\right) \ldots\left(z+p+m+N_{\mathscr{I}}\right)} z^{m}
$$

then clearly $g_{p, m}$ approaches $z^{m}$ in $E$ as $p \rightarrow \infty$. On the other hand, there is a partial fractions decomposition of $g_{p, m}$ into a linear combination of elements of $\left\{f_{j}\right\}$, thus establishing the claim.

Now $f_{j}$ is the Laplace transform of $e^{-j s}$; so if

$$
\varphi_{\beta, j}(s)=\frac{e^{-j s}}{s^{\beta-1 / 2} \vartheta_{\mathscr{A}}(s)}
$$

then $\mathscr{T}_{\mathscr{I}, \beta} \varphi_{\beta, j}=f_{j}$. However in general (that is, for $\beta \geqslant 1$ ), $\varphi_{\beta, j}$ does not belong to $L^{2}\left(m_{\mathscr{I}}\right)$, so that $f_{j}$ is not in $\mathscr{H}_{\mathscr{I}, \beta}$.

Nevertheless, if $\varphi_{\beta, j, n}:=\varphi_{\beta, j} \chi_{[1 / n, \infty)}$ then it is readily verified that $\varphi_{\beta, j, n} \in L^{2}\left(m_{\mathscr{I}}\right)$ for all $\beta>\max \left(0, \theta_{\mathscr{I}}\right)$. Therefore $f_{\beta, j, n}:=\mathscr{T}_{\mathscr{I}, \beta} \varphi_{\beta, j, n} \in \mathscr{H}_{\mathscr{I}, \beta}$. Now $f_{\beta, j, n}(z)=e^{-(z+j) / n} /(z+j)$, so that $f_{\beta, j, n} \in E$ (and in fact does not depend on $\beta$ ). Clearly $f_{\beta, j, n}$ converges to $f_{j}$ in $E$ as $n \rightarrow \infty$. So the linear span of $\left\{f_{\beta, j, n}\right\}_{j>N_{\mathcal{I}}, n \in \mathbb{N}}$ is $E$-dense in the linear span of $\left\{f_{j}\right\}_{j>N_{\mathscr{I}}}$, which itself is $E$-dense in $E$, and we have finished. 
Theorem 3. Let $\mathscr{I} \in \mathbb{N}$ be non-empty, and $\operatorname{Re}(\beta)>\max \left(0, \theta_{\mathscr{I}}\right)$. Suppose the open disc $D$ satisfies $[0,1] \subset D, \bar{D} \subset R_{\mathscr{I}}$, and $\bar{D} \subset \operatorname{int}\left(\bigcap_{n \in \mathscr{I}} S_{n}(D)\right)$, where $S_{n}(z)=1 / z-n$.

(a) If $E$ denotes any of the spaces $A_{\infty}(D), H^{2}(D), C^{\omega}[0,1]$, or $C^{\infty}[0,1]$, then $\sigma\left(\mathscr{L}_{\mathscr{I}, \beta} \mid E\right)=\sigma\left(\mathscr{L}_{\mathscr{I}, \beta} \mid \mathscr{H}_{\mathscr{I}, \beta}\right)$. If furthermore $\beta$ is real, then $\sigma\left(\mathscr{L}_{\mathscr{I}, \beta} \mid E\right)$ is real.

(b) If $E$ denotes either of the spaces $C^{k}[0,1]($ for $k \in \mathbb{N})$ or $C^{\alpha}[0,1]$ (for $0<\alpha \leqslant 1)$, then the non-essential spectrum of $\mathscr{L}_{\mathscr{I}, \beta}: E \rightarrow E$ is a subset of $\sigma\left(\mathscr{L}_{\mathscr{I}, \beta} \mid \mathscr{H}_{\mathscr{I}, \beta}\right)$. If furthermore $\beta$ is real, then the non-essential spectrum of $\mathscr{L}_{\mathscr{I}, \beta}: E \rightarrow E$ is real.

Proof. Grabiner's intertwining theorem [17], which was initially formulated in the context of Banach spaces, has been extended by Bandtlow [4, Theorem A.6.2] to more general topological vector spaces. This extension asserts the following. Suppose $E$ is a sequentially complete and barrelled topological vector space, with a dense subspace $F$. Suppose $F$ carries a topology stronger than the one induced by $E$, and that $F$ is itself sequentially complete and barrelled with respect to this topology. If both $E$ and $F$ are invariant under a continuous linear operator $L$, then the discrete part of $\sigma(L \mid E)$ is a subset of the discrete part of $\sigma(L \mid F)$. Moreover, if both $L: E \rightarrow E$ and $L: F \rightarrow F$ have countable spectrum, then these spectra coincide.

We choose $L=\mathscr{L}_{\mathscr{J}, \beta}$ and $F=\mathscr{H}_{\mathscr{I}, \beta}$. If $E$ is any of the spaces in the statement of the theorem then $F$ is continuously and densely embedded in $E$ by Lemma 6 . Part (b) therefore follows from Bandtlow's intertwining theorem, and from Theorem 2 when $\beta$ is real. If now $E$ is any of the spaces in the statement of part (a), then we recall from $\S 3$ that $\sigma\left(\mathscr{L}_{\mathscr{I}, \beta} \mid E\right)$ is countable. Therefore (a) follows from the intertwining theorem as well, and from Theorem 2 when $\beta$ is real.

In fact it is possible to say a little more about any $C^{\infty}$ eigenfunction of $\mathscr{L}_{\mathscr{Y}, \beta}$ corresponding to a non-zero eigenvalue, as described in the following corollary.

Corollary 1. Let $\mathscr{I} \subset \mathbb{N}$ be non-empty, and $\operatorname{Re}(\beta)>\max \left(0, \theta_{\mathscr{I}}\right)$. Every $C^{\infty}$ eigenfunction corresponding to a non-zero eigenvalue of $\mathscr{L}_{\mathscr{I}, \beta}$ is holomorphic in the cut plane $\mathbb{C} \backslash(-\infty,-1]$.

Proof. Let $A$ denote the restriction of $\mathscr{L}_{\mathscr{I}, \beta}$ to $\mathscr{H}_{\mathscr{I}, \beta}$, and let $B$ denote the restriction of $\mathscr{L}_{\mathscr{Y}, \beta}$ to $C^{\infty}[0,1]$. By Theorem 3 we know that every eigenvalue $\lambda$ of $B$ is also an eigenvalue of $A$. If $\lambda \neq 0$ then it is an isolated element of both spectra.

Let us suppose that the geometric multiplicity of $\lambda$ as an eigenvalue of $A$ coincides with its geometric multiplicity as an eigenvalue of $B$. Then any $C^{\infty}$ eigenfunction $f$ corresponding to $\lambda$ is actually an element of $\mathscr{H}_{\mathscr{I}, \beta}$. Since $\lambda \neq 0$, the eigenequation can be written $\lambda^{-1} \mathscr{L}_{\mathscr{I}, \beta} f=f$, and iterated to give $\lambda^{-n} \mathscr{L}_{\mathscr{I}, \beta}^{n} f=f$ for all $n \geqslant 0$. But $f$ is holomorphic in the half-plane $R_{\mathscr{I}}$, from which we successively deduce that $\lambda^{-n} \mathscr{L}_{\mathscr{I}, \beta}^{n} f=f$ is holomorphic in some region $\Omega_{n}$, where $\Omega_{0} \subset \Omega_{1} \subset \ldots$ and $\bigcup_{n=0}^{\infty} \Omega_{n}=\mathbb{C} \backslash(-\infty,-1]$. Since $\lambda$ was an arbitrary non-zero eigenvalue, and $f$ an arbitrary eigenfunction, it follows that every $C^{\infty}$ eigenfunction for a non-zero eigenvalue is holomorphic in the cut plane $\mathbb{C} \backslash(-\infty,-1]$, as required.

It remains to show that indeed the geometric multiplicity of $\lambda$ as an eigenvalue 
of $A$ coincides with its geometric multiplicity as an eigenvalue of $B$. For the following argument we are grateful to O. F. Bandtlow, who pointed out its omission in our original proof (see also [5] for a thorough investigation of related questions).

If $\iota: \mathscr{H}_{\mathscr{I}, \beta} \rightarrow C^{\infty}[0,1]$ denotes the inclusion map, then the relation $B \circ \iota=$ $\iota \circ A$ gives

$$
(z-B)^{-1} \circ \iota=\iota \circ(z-A)^{-1}
$$

for all $z$ belonging to the resolvent set of both $A$ and $B$.

Allan's functional calculus [1] applies to continuous linear operators $L$ on a certain class of locally convex topological vector spaces which includes $C^{\infty}[0,1]$. This generalises Taylor's functional calculus for closed operators on Banach spaces, and asserts that, under suitable hypotheses (see [1, Theorem 5.3]),

$$
F(L)=\frac{1}{2 \pi i} \int_{\partial C} \frac{F(z)}{z-L} d z
$$

for functions $F$ holomorphic in a neighbourhood of the spectrum of $L$, where $C$ is a suitable Cauchy domain (see [1, Definition 5.2]). Combining this with (7) we can derive the relations

$$
\iota \circ \Pi(\lambda ; A)=\Pi(\lambda ; B) \circ \iota
$$

and

$$
\iota \circ A \circ \Pi(\lambda ; A)=\Pi(\lambda ; B) \circ B \circ \iota,
$$

where $\Pi(\lambda ; A)$ and $\Pi(\lambda ; B)$ denote the respective spectral projections at $\lambda$.

From (8) it easily follows that

$$
\iota(\mathscr{R}(\Pi(\lambda ; A))) \subset \mathscr{R}(\Pi(\lambda ; B)),
$$

where $\mathscr{R}(\Pi(\lambda ; A))$ and $\mathscr{R}(\Pi(\lambda ; B))$ are the respective generalised eigenspaces corresponding to $\lambda$. Now Proposition A.6.5 of [4] implies that $\mathscr{R}(\Pi(\lambda ; A))$ and $\mathscr{R}(\Pi(\lambda ; B))$ have the same dimension (that is, the algebraic multiplicity of $\lambda$ is the same for both $A$ and $B)$. Now $\mathscr{K}_{\mathscr{I}, \beta}: L^{2}\left(m_{\mathscr{I}}\right) \rightarrow L^{2}\left(m_{\mathscr{I}}\right)$ is trace class by Proposition 5, and hence so is the operator $A$, by Theorem 1(b). In particular, $A$ is compact, so that $\mathscr{R}(\Pi(\lambda ; A))$ is finite dimensional [15, Theorem 7.4.5]. Therefore equation (10), together with the injectivity of $\iota$, implies that $\iota$ is an isomorphism between the finite-dimensional generalised eigenspaces $\mathscr{R}(\Pi(\lambda ; A))$ and $\mathscr{R}(\Pi(\lambda ; B))$.

Using (9), and the fact that $B$ commutes with $\Pi(\lambda ; B)$, we see that the restrictions $B \mid \mathscr{R}(\Pi(\lambda ; B))$ and $A \mid \mathscr{R}(\Pi(\lambda ; A))$ are similar, so that their Jordan decompositions are identical. In particular,

$$
\text { nullity }[(\lambda-A) \mid \mathscr{R}(\Pi(\lambda ; A))]=\operatorname{nullity}[(\lambda-B) \mid \mathscr{R}(\Pi(\lambda ; B))] \text {. }
$$

To complete the proof, we observe that since $\Pi(\lambda ; A)$ and $\Pi(\lambda ; B)$ are spectral projections then

$$
\operatorname{ker}(\lambda-A)=\operatorname{ker}(A \mid \mathscr{R}(\Pi(\lambda ; A))) \quad \text { and } \quad \operatorname{ker}(\lambda-B)=\operatorname{ker}(B \mid \mathscr{R}(\Pi(\lambda ; B))) .
$$

Hence nullity $(\lambda-A)=\operatorname{nullity}(\lambda-B)$, as required.

REmark 4. Suppose that $\mathscr{I}$ is a singleton $\mathscr{I}=\{n\}$, and $\beta \in \mathbb{C}$ is arbitrary. In this case $\mathscr{L}_{\mathscr{I}, \beta}$ is just a weighted composition operator

$$
\mathscr{L}_{\mathscr{I}, \beta} f(z)=(n+z)^{-2 \beta} f(1 /(n+z)),
$$


whose spectrum can be determined explicitly (cf. [27, 32]). If

$$
z_{n}=\frac{1}{n+\frac{1}{n+\ldots}}
$$

is the fixed point of $1 /(n+z)$, then

$$
\sigma\left(\mathscr{L}_{\mathscr{I}, \beta} \mid C^{\infty}[0,1]\right)=\left\{(-1)^{k} z_{n}^{2 k+2 \beta}: k \in \mathbb{Z}_{\geqslant 0}\right\} \cup\{0\} .
$$

7. Analytic properties of $\beta \mapsto \mathscr{L}_{\mathscr{I}, \beta}$

In this section we consider the analytic properties of the map $\beta \mapsto \mathscr{L}_{\mathscr{J}, \beta}$, where $\mathscr{L}_{\mathscr{I}, \beta}$ is considered as a trace class operator on a suitable Hardy space $H^{2}(D)$. In particular, $\mathscr{L}_{\mathscr{I}, \beta}$ is an element of the complex Banach space $B\left(H^{2}(D)\right)$ of bounded linear operators from $H^{2}(D)$ to itself. The analyticity of a map from some complex domain into $B\left(H^{2}(D)\right)$ is then understood (cf. [46, p. 205]) to mean the existence of a derivative at every point.

The map $\beta \mapsto \mathscr{L}_{\mathscr{I}, \beta}$ is certainly holomorphic in the half-plane $\operatorname{Re}(\beta)>\theta_{\mathscr{I}}$, and for general alphabets $\mathscr{I} \subset \mathbb{N}$ there is no reason to expect an analytic continuation to a larger region. However for alphabets of an arithmetic nature, such as those studied in [31], such continuations are often possible, as will be described in this section. The original result in this direction is due to Mayer [33], and asserts that $\beta \mapsto \mathscr{L}_{\mathbb{N}, \beta}$ extends meromorphically to the entire plane, the only poles being simple ones at $\beta=\frac{1}{2}(1-k)$, where $k \in \mathbb{Z}_{\geqslant 0}$. The notion of analyticity used by Mayer is weaker than the one we consider here; in [33] it means that $\beta \mapsto \mathscr{L}_{\mathscr{I}, \beta} f(z)$ is analytic for every $z \in D$ and $f \in H^{2}(D)$.

THEOREM 4. Let $\mathscr{I} \subset \mathbb{N}$ be non-empty. Let $D \subset \mathbb{C}$ be an open disc with $[0,1] \subset D, \bar{D} \subset R_{\mathscr{I}}$, and such that $\bar{D} \subset \operatorname{int}\left(\bigcap_{n \in \mathscr{I}} S_{n}(D)\right)$, where $S_{n}(z)=1 / z-n$.

Let $\zeta_{\mathscr{I}}(s, z)=\sum_{n \in \mathscr{I}}(z+n)^{-s}$ denote the Hurwitz zeta function for the alphabet $\mathscr{I}$. If the map $\beta \mapsto \zeta_{\mathscr{I}}(2 \beta, z)$ has an analytic continuation to a complex domain $U$, then the following hold.

(a) The map $\beta \mapsto \mathscr{L}_{\mathscr{I}, \beta}$ also has an analytic continuation to $U$.

(b) The singularities of the analytic continuation of $\beta \mapsto \mathscr{L}_{\mathscr{J}, \beta}$ are at the points $\frac{1}{2}(p-k)$ with $k \in \mathbb{Z}_{\geqslant 0}$, where $p$ is a singularity of $s \mapsto \zeta_{\mathscr{A}}(s, z)$.

(c) If $\bar{U}=\mathbb{C}$, and the analytic continuation of $\beta \mapsto \zeta_{\mathscr{A}}(2 \beta, z)$ to $U$ is a meromorphic one to $\bar{U}=\mathbb{C}$, then $\beta \mapsto \mathscr{L}_{\mathscr{I}, \beta}$ also has a meromorphic continuation to $\mathbb{C}$, with poles of order $m$ at the points $\frac{1}{2}(p-k)$ with $k \in \mathbb{Z} \geqslant 0$, where $p$ is a pole of order $m$ of $s \mapsto \zeta_{\mathscr{I}}(s, z)$.

(d) If $s \mapsto \zeta_{\mathscr{I}}(s, z)$ has an algebraic or logarithmic branch singularity at the point $p$, then $\beta \mapsto \mathscr{L}_{\mathscr{J}, \beta}$ has algebraic or logarithmic, respectively, branch singularities at those points $\frac{1}{2}(p-k)$ with $k \in \mathbb{Z} \geqslant 0$ which lie in $\bar{U}$.

(e) For each $\beta \in U, \mathscr{L}_{\mathscr{I}, \beta}: H^{2}(D) \rightarrow H^{2}(D)$ is a trace class operator.

Proof. We follow Mayer [33] in expressing $\beta \mapsto \mathscr{L}_{\mathscr{I}, \beta}$ as the sum of two maps, the first of which is meromorphic with range the class of rank- $(N+1)$ operators, the second of which is entire and trace class-valued. We will then let $N \rightarrow \infty$. 
Define the operators $\pi_{k}, \mathscr{P}_{N}: H^{2}(D) \rightarrow H^{2}(D)$ by

$$
\pi_{k} f(z)=\frac{f^{(k)}(0)}{k !} z^{k}, \quad \mathscr{P}_{N}=\sum_{k=0}^{N} \pi_{k} .
$$

Letting $\mathscr{Q}_{N}=I-\mathscr{P}_{N}$ we write, for any $N \in \mathbb{Z}_{\geqslant 0}$,

$$
\mathscr{L}_{\mathscr{I}, \beta}=\mathscr{L}_{\mathscr{I}, \beta} \circ \mathscr{P}_{N}+\mathscr{L}_{\mathscr{I}, \beta} \circ \mathscr{Q}_{N} .
$$

Each of the rank-1 projections $\mathscr{L}_{\mathscr{I}, \beta} \circ \pi_{k}$ can be expressed as

$$
\mathscr{L}_{\mathscr{I}, \beta} \pi_{k} f(z)=\frac{f^{(k)}(0)}{k !} \zeta_{\mathscr{I}}(2 \beta+k, z)
$$

for $f \in H^{2}(D), \operatorname{Re}(\beta)>\frac{1}{2}\left(\theta_{\mathscr{I}}-k\right)$, and for $z \in D^{\prime}$, some open disc concentric with $D$ and of strictly larger radius, as in Proposition 1.

Fix $f \in H^{2}(D)$ and $z \in D^{\prime}$. Let $P$ denote the set of singularities of $s \mapsto \zeta_{\mathscr{I}}(s, z)$. Since $\beta \mapsto \zeta_{\mathscr{I}}(2 \beta, z)$ extends analytically to $U$, with singularities in $\frac{1}{2} P$, then $\beta \mapsto \mathscr{L}_{\mathscr{I}, \beta} \pi_{k} f(z)$ extends to the translated region $U-k$, with singularities translated to lie in the set $\frac{1}{2}(P-k) \cap \bar{U}$. Therefore $\beta \mapsto \mathscr{L}_{\mathscr{I}, \beta} \mathscr{P}_{N} f(z)$ extends analytically to $U$, with singularities in the set $\left\{\frac{1}{2}(p-k): 0 \leqslant k \leqslant N, p \in P\right\} \cap \bar{U}$.

Now we claim that for arbitrary fixed $f \in H^{2}(D)$ and $z \in D^{\prime}$, the map $\beta \mapsto \mathscr{L}_{\mathscr{I}, \beta} \mathscr{Q}_{N} f(z)$ can be extended holomorphically to the half-plane

$$
\operatorname{Re}(\beta)>\frac{1}{2}\left(\theta_{\mathscr{I}}-N-1\right) \text {. }
$$

If $\mathscr{I}$ is finite then this claim is clearly true, so suppose $\mathscr{I}$ is infinite, and write $\mathscr{I}=\left\{n_{k}\right\}_{k=1}^{\infty}$, where $n_{1}<n_{2}<\ldots$.

If $\xi>0$ is sufficiently small, then every $f \in H^{2}(D)$ has a power series expansion around 0 , uniformly convergent for $|z|<\xi$. It follows from Cauchy's theorem that there is a constant $C_{f}>0$ such that

for all $|z|<\xi$.

$$
\left|\mathscr{Q}_{N} f(z)\right| \leqslant C_{f}|z|^{N+1}
$$

Since $\mathscr{I}=\left\{n_{k}\right\}$ is infinite, we may choose $m \in \mathbb{N}$ large enough so that

We now write

$$
\left|\frac{1}{z+n_{k}}\right|<\xi \quad \text { for any } z \in D^{\prime} \text { and } k>m \text {. }
$$

$$
\begin{aligned}
\mathscr{L}_{\mathscr{I}, \beta} \mathscr{Q}_{N} f(z)= & \sum_{k=1}^{m}\left(\frac{1}{z+n_{k}}\right)^{2 \beta} \mathscr{Q}_{N} f\left(\frac{1}{z+n_{k}}\right) \\
& +\sum_{k=m+1}^{\infty}\left(\frac{1}{z+n_{k}}\right)^{2 \beta} \mathscr{Q}_{N} f\left(\frac{1}{z+n_{k}}\right) .
\end{aligned}
$$

The first summation in this expression is finite, and so can be extended to an entire function of $\beta$. Using (12) we see that the second summation is absolutely and uniformly bounded on $D^{\prime}$ by a constant multiple of $\sum_{n \in \mathscr{I}} n^{-2 \operatorname{Re}(\beta)-N-1}$, which in particular is convergent for $\operatorname{Re}(\beta)>\frac{1}{2}\left(\theta_{\mathscr{I}}-N-1\right)$, and therefore defines a holomorphic function in this half-plane.

Therefore for any fixed $f \in H^{2}(D)$ and $z \in D^{\prime}$, the map

$$
\beta \mapsto \mathscr{L}_{\mathscr{I}, \beta} f(z)=\mathscr{L}_{\mathscr{I}, \beta} \mathscr{P}_{N} f(z)+\mathscr{L}_{\mathscr{I}, \beta} \mathscr{Q}_{N} f(z)
$$


has an analytic extension to $U \cap\left\{\beta \in \mathbb{C}: \operatorname{Re}(\beta)>\frac{1}{2}\left(\theta_{\mathscr{I}}-N-1\right)\right\}$, for any $N \in \mathbb{Z}_{\geqslant 0}$. Letting $N \rightarrow \infty$ shows that $\beta \mapsto \mathscr{L}_{\mathscr{I}, \beta} f(z)$ has an analytic extension to $U$. The nature of any singularity of $\beta \mapsto \mathscr{L}_{\mathscr{I}, \beta} f(z)$ is clearly the same as the corresponding singularity of $s \mapsto \zeta_{\mathscr{T}}(s, z)$.

We now claim that $\beta \mapsto \mathscr{L}_{\mathscr{I}, \beta}$ is weakly analytic on $U$; that is, given any $f \in H^{2}(D)$ and any bounded linear functional $l \in H^{2}(D)^{\prime}$, the map $\beta \mapsto l\left(\mathscr{L}_{\mathscr{G}, \beta} f\right)$ has an analytic extension to $U$. Every functional has a representation $l=(\cdot, g)$, for some $g \in H^{2}(D)$, so that

$$
l\left(\mathscr{L}_{\mathscr{I}, \beta} f\right)=\frac{1}{2 \pi} \int_{0}^{2 \pi} \mathscr{L}_{\mathscr{I}, \beta} f\left(c+r e^{i t}\right) \overline{g\left(c+r e^{i t}\right)} d t,
$$

where $c$ denotes the centre of $D$, and $r$ denotes its radius. We have shown that for every $t \in[0,2 \pi)$, the map $\beta \mapsto \mathscr{L}_{\mathscr{J}, \beta} f\left(c+r e^{i t}\right)$ is holomorphic. Moreover, on any compact subset $K$ of $U$, the integral (13) is uniformly convergent for $\beta \in K$. The analyticity of $\beta \mapsto l\left(\mathscr{L}_{\mathscr{I}, \beta} f\right)$ then follows from Lemma 1.1 of [25].

The weak analyticity of $\beta \mapsto \mathscr{L}_{\mathscr{I}, \beta}$ in fact implies strong analyticity (see [46, Theorem 4.4-F]); that is, for any $f \in H^{2}(D)$ the map $\beta \mapsto \mathscr{L}_{\mathscr{I}, \beta} f$ is analytic on $U$ (as an $H^{2}(D)$-valued map). But strong analyticity in turn implies the analyticity of $\beta \mapsto \mathscr{L}_{\mathscr{I}, \beta}$ as a $B\left(H^{2}(D)\right)$-valued map (see [46, Theorem 4.4-G]), which is the result we want. Once again the nature of any singularity of $\beta \mapsto \mathscr{L}_{\mathscr{J}, \beta}$ is clearly the same as the corresponding singularity of $s \mapsto \zeta_{\mathscr{I}}(s, z)$.

The operator $\mathscr{L}_{\mathscr{I}, \beta} \circ \mathscr{Q}_{N}$ is trace class for each $\beta \in U$, by the same calculations as in Proposition 1. Adding the finite rank operator $\mathscr{L}_{\mathscr{I}, \beta} \circ \mathscr{P}_{N}$ gives the analytically continued operator $\mathscr{L}_{\mathscr{I}, \beta}$, which is therefore itself trace class for each $\beta \in U$.

THeOREM 5. Let $\mathscr{I} \subset \mathbb{N}$ be non-empty. Let $D \subset \mathbb{C}$ be an open disc with $[0,1] \subset D$ and $\bar{D} \subset R_{\mathscr{I}}$, and such that $\bar{D} \subset \operatorname{int}\left(\bigcap_{n \in \mathscr{I}} S_{n}(D)\right)$, where $S_{n}(z)=1 / z-n$. Define $\mathscr{L}_{\mathscr{I}, \beta}: H^{2}(D) \rightarrow H^{2}(D)$ by (5) on the half-plane $\operatorname{Re}(\beta)>\theta_{\mathscr{g}}$. For the following alphabets $\mathscr{I}$, the map $\beta \mapsto \mathscr{L}_{\mathscr{I}, \beta}$ has an analytic continuation to the region stated, and in each case $\mathscr{L}_{\mathscr{I}, \beta}$ is a trace class operator for every $\beta$ in the extended region.

(i) $\mathscr{I}$ finite (and non-empty); $\beta \mapsto \mathscr{L}_{\mathscr{Y}, \beta}$ is an entire function of $\beta$.

(ii) $\mathscr{I}=a \mathbb{N}+b$ for $a, b \in \mathbb{N} ; \beta \mapsto \mathscr{L}_{\mathscr{I}, \beta}$ extends meromorphically to $\mathbb{C}$ with simple poles at $\beta=\frac{1}{2}(1-k)$ for $k \in \mathbb{Z}_{\geqslant 0}$.

(iii) $\mathscr{I}=\left\{n^{a}\right\}_{n=1}^{\infty}$ for $a \in \mathbb{N} ; \quad \beta \mapsto \mathscr{L}_{\mathscr{I}, \beta}$ extends meromorphically to the complex plane with simple poles at $\beta=\frac{1}{2}(1 / a-k)$ for $k \in \mathbb{Z} \geqslant 0$.

(iv) $\mathscr{I}=\{\psi(n)\}_{n=1}^{\infty}$ with $\psi \in \mathbb{N}[z]$ a polynomial of degree $d>1$. Then $\theta_{\mathscr{I}}=1 / 2 d$, and $\beta \mapsto \mathscr{L}_{\mathscr{I}, \beta}$ extends meromorphically to $\mathbb{C}$, the only poles being simple ones among the points $\beta=(1-k) / 2 d$ with $k \in \mathbb{Z} \geqslant 0$.

(v) $\mathscr{I}=\left\{a^{n}\right\}_{n=1}^{\infty}$ for $a \in \mathbb{Z}_{\geqslant 2} ; \beta \mapsto \mathscr{L}_{\mathscr{I}, \beta}$ extends meromorphically to the complex plane with poles at $\left\{-\frac{1}{2} k+(\pi i / \log a) m:(k, m) \in \mathbb{Z} \geqslant_{0} \times \mathbb{Z}\right\}$.

(vi) $\mathscr{I}=\{$ prime numbers $\}$. Then $\theta_{\mathscr{I}}=\frac{1}{2}$. There is a sequence $\left\{\beta_{r}\right\}_{r=1}^{\infty}$, with $\beta_{1}=\frac{1}{2}$, whose set of accumulation points is the imaginary axis $\operatorname{Re}(\beta)=0$, such that $\beta \mapsto \mathscr{L}_{\mathscr{I}, \beta}$ extends analytically to a domain $\{\beta \in \mathbb{C}: \operatorname{Re}(\beta)>0\} \backslash \bigcup_{r=1}^{\infty} L_{r}$, where $L_{r}=\left\{\frac{1}{2} \beta_{r}-t: t \geqslant 0\right\}$. The map has a logarithmic singularity at each point $\beta_{r}$.

(vii) $\mathscr{I}$ the set of integers which are the sum of two squares (of natural numbers). Then $\theta_{\mathscr{I}}=\frac{1}{2}$, and $\mathscr{L}_{\mathscr{I}, \beta}=\left(\beta-\frac{1}{2}\right)^{-1 / 2} \mathscr{M}_{\mathscr{I}, \beta}$, where $\beta \mapsto \mathscr{M}_{\mathscr{I}, \beta}$ extends 
analytically to a domain $\left\{\beta \in \mathbb{C}: \operatorname{Re}(\beta)>\frac{1}{2}-\frac{1}{2} A[\log (2|\operatorname{Im}(\beta)|+2)]^{-A}\right\}$ for some $A>0$.

(viii) $\mathscr{I}=\left\{n_{k}\right\}_{k=1}^{\infty}$ is such that there exists $\gamma>0$ with $n_{k+1} \geqslant n_{k}^{1+\gamma}$ for all $k$. Then $\theta_{\mathscr{I}}=0$, and the line $\operatorname{Re}(\beta)=0$ is a natural boundary for the map $\beta \mapsto \mathscr{L}_{\mathscr{I}, \beta}$.

Proof. (i) This is clear.

By Theorem 4 , the analytic properties of $\beta \mapsto \mathscr{L}_{\mathscr{I}, \beta}$ are closely related to those of $s \mapsto \zeta_{\mathscr{T}}(s, z)$. In case (ii) we can exploit this directly.

(ii) We can write

$$
\zeta_{\mathscr{A}}(s, z)=\sum_{n=1}^{\infty}\left(\frac{1}{a n+b+z}\right)^{s}=a^{-s} \zeta_{\mathbb{N}}\left(s, \frac{z+b}{a}\right) .
$$

If $\zeta(s, z)=\sum_{n=0}^{\infty}(z+n)^{-s}$ denotes the classical Hurwitz zeta function, then we have

$$
\zeta_{\mathbb{N}}\left(s, \frac{z+b}{a}\right)=\zeta\left(s, \frac{z+b}{a}\right)-\left(\frac{a}{z+b}\right)^{s} .
$$

Now $\zeta(s,(z+b) / a)$ has a meromorphic extension to the entire complex plane, with a simple pole at $s=1\left[6\right.$, p. 502], so the same is true of $\zeta_{\mathbb{N}}(s,(z+b) / a)$, and hence of $\zeta_{\mathscr{I}}(s, z)$. The result then follows by Theorem 4 .

In cases (iii)-(viii) it will be more convenient to relate $\zeta_{\mathscr{I}}(s, z)$ to $\zeta_{\mathscr{I}}(s)$. The analytic properties of this latter zeta function are known for the alphabets $\mathscr{I}$ considered here.

We claim that if $\zeta_{\mathscr{I}}(s)$ has an analytic extension to a complex domain $U$, with set of singularities $Q$, then for $z \in D$, the map $s \mapsto \zeta_{\mathscr{I}}(s, z)$ has an analytic extension to the same domain $U$, and that its singularities are contained in $\{q-k: q \in Q, k \in \mathbb{Z} \geqslant 0\} \cap \bar{U}$.

Let us prove this claim. Write $\mathscr{I}=\left\{n_{k}\right\}_{k=1}^{\infty}$, where $n_{1}<n_{2}<\ldots$. We can choose $m \in \mathbb{N}$ sufficiently large to ensure that the closure of $D$ is contained in the disc of radius $\frac{1}{2} n_{m}$ centred at 0 . Define

$$
g(s, z)=\sum_{k=1}^{m-1}\left(\frac{1}{z+n_{k}}\right)^{s}, \quad h(s, z)=\zeta_{\mathscr{I}}(s, z)-g(s, z),
$$

for $z \in D$ and $\operatorname{Re}(s)>2 \theta_{\mathscr{I}}$. For fixed $z \in D$, the map $s \mapsto g(s, z)$ is clearly entire. We will consider the analytic extension of $s \mapsto h(s, z)$. Let $\mathscr{J}=\left\{n_{k}\right\}_{k=m}^{\infty}$ be the tail of the alphabet $\mathscr{I}$. For $z \in D$ and $\operatorname{Re}(s)>2 \theta_{\mathscr{I}}$ we have

$$
\begin{aligned}
h(s, z) & =\sum_{k=m}^{\infty} \frac{1}{n_{k}^{s}}\left(\frac{1}{1+z / n_{k}}\right)^{s} \\
& =\sum_{k=m}^{\infty} \frac{1}{n_{k}^{s}} \sum_{l=0}^{\infty}\left(\begin{array}{c}
-s \\
l
\end{array}\right)\left(-\frac{z}{n_{k}}\right)^{l} \\
& =\sum_{l=0}^{\infty}\left(\begin{array}{c}
-s \\
l
\end{array}\right)(-z)^{l} \sum_{k=m}^{\infty} \frac{1}{n_{k}^{s+l}} \\
& =\sum_{l=0}^{\infty}\left(\begin{array}{c}
-s \\
l
\end{array}\right)(-z)^{l} \zeta_{\mathscr{J}}(s+l),
\end{aligned}
$$


where the interchange of order of summation is clearly justified, and where

$$
\left(\begin{array}{c}
-s \\
l
\end{array}\right)=\frac{\Gamma(1-s)}{\Gamma(l+1) \Gamma(-s-l+1)}
$$

(so in particular if $-s \in \mathbb{N}$ with $-s<l$ then $\left(\begin{array}{c}-s \\ l\end{array}\right)=0$ ).

We will prove that the map

$$
s \mapsto \sum_{l=N}^{\infty}\left(\begin{array}{c}
-s \\
l
\end{array}\right)(-z)^{l} \zeta_{\mathscr{J}}(s+l)
$$

extends analytically to $U \cap\left\{s \in \mathbb{C}: \operatorname{Re}(s)>2 \theta_{\mathscr{I}}-N\right\}$, for all $N \in \mathbb{N}$. This will complete the proof of the claim, since it is easily seen that

$$
s \mapsto \sum_{l=0}^{N-1}\left(\begin{array}{c}
-s \\
l
\end{array}\right)(-z)^{l} \zeta_{\mathscr{J}}(s+l)
$$

extends analytically to $U$, with singularities in the set

$$
\{q-k: q \in Q, 0 \leqslant k \leqslant N-1\} \cap \bar{U} .
$$

Fix $N \in \mathbb{N}$ and set $h_{N}(s, w)=\sum_{l=N}^{\infty}\left(\begin{array}{c}-s \\ l\end{array}\right)(-w)^{l}$. By a calculation analogous to the one above we have

$$
\sum_{l=N}^{\infty}\left(\begin{array}{c}
-s \\
l
\end{array}\right)(-z)^{l} \zeta_{\mathscr{J}}(s+l)=\sum_{k=m}^{\infty} \frac{1}{n_{k}^{s}} h_{N}\left(s, \frac{z}{n_{k}}\right) .
$$

Let $K$ be any compact subset of the half-plane $\operatorname{Re}(s)>2 \theta_{\mathscr{I}}-N$. By the Cauchy formula there is a constant $A>0$ such that $\left|h_{N}(s, w)\right| \leqslant A|w|^{N}$ for $s \in K$ and $|w| \leqslant \frac{1}{2}$. Now if $z \in D$ then $\left|z / n_{k}\right| \leqslant \frac{1}{2}$ for all $k \geqslant m$. Therefore

$$
\left|\sum_{k=m}^{\infty} \frac{1}{n_{k}^{s}} h_{N}\left(s, \frac{z}{n_{k}}\right)\right| \leqslant A \max _{z \in D}|z|^{N} \sum_{k=m}^{\infty}\left(\frac{1}{n_{k}}\right)^{\operatorname{Re}(s)+N},
$$

for all $z \in D$ and $s \in K$.

The right-hand side of this inequality is finite, since $\operatorname{Re}(s)>2 \theta_{\mathscr{I}}-N$, so indeed the map

$$
s \mapsto \sum_{l=N}^{\infty}\left(\begin{array}{c}
-s \\
l
\end{array}\right)(-z)^{l} \zeta_{\mathscr{J}}(s+l)=\sum_{k=m}^{\infty} \frac{1}{n_{k}^{s}} h_{N}\left(s, \frac{z}{n_{k}}\right)
$$

is holomorphic on $\operatorname{Re}(s)>2 \theta_{\mathscr{I}}-N$, and the claim is proved. The claim will now be used to establish parts (iii)-(viii) of the theorem.

(iii) In this case we have $\zeta_{\mathscr{I}}(s)=\zeta(a s)$, where $\zeta$ is Riemann's zeta function, which has a meromorphic extension to all of $\mathbb{C}$, with a simple pole at $s=1$. Therefore $\zeta_{\mathscr{I}}(s)$ has a meromorphic extension to the whole plane, with a simple pole at $s=1 / a$. The above claim means that $\zeta_{\mathscr{I}}(s, z)$ has a meromorphic continuation to $\mathbb{C}$, with simple poles at $s=1 / a-k$ with $k \in \mathbb{Z}_{\geqslant 0}$. (In the case $p=1$ all these poles except for $k=0$ are cancelled by zeros.) The result follows by Theorem 4 .

(iv) The function $\zeta_{\mathscr{I}}(s)$ has a meromorphic extension to all of $\mathbb{C}$, with a simple pole at $s=1 / d$, and possible further simple poles at $s=-k / d$ for $k \in \mathbb{Z} \geqslant 0[36]$. By the above claim, the same is true of $\zeta_{\mathscr{I}}(s, z)$, and the result then follows by Theorem 4 . 
(v) Now

$$
\zeta_{\mathscr{I}}(s)=\sum_{m=1}^{\infty} a^{-m s}=\frac{1}{1-a^{-s}},
$$

which extends meromorphically to $\mathbb{C}$ with simple poles at $s=(2 \pi i / \log a) m$ for $m \in \mathbb{Z}$. The above claim implies that $\zeta(s, z)$ has a meromorphic continuation to $\mathbb{C}$, with simple poles at points $s=(2 \pi i / \log a) m-k$ for $k \in \mathbb{Z} \geqslant 0$ and $m \in \mathbb{Z}$. The result then follows by Theorem 4 .

(vi) For $\mathscr{I}=\{$ primes $\}$, the Dirichlet series $\zeta_{\mathscr{I}}(s)$ is holomorphic for $\operatorname{Re}(s)>1$, has a logarithmic singularity at $s=1$, and can be continued into the half-plane $\operatorname{Re}(s)>0$ with logarithmic singularities at points $s_{r}$ which accumulate on the imaginary axis and do not accumulate elsewhere [28]. Removing suitable half-lines, for example $L_{r}^{\prime}=\left\{s_{r}-t: t \geqslant 0\right\}$, gives a domain $\{s \in \mathbb{C}: \operatorname{Re}(s)>0\} \backslash \bigcup_{r=1}^{\infty} L_{r}^{\prime}$ on which $\zeta_{\mathscr{I}}(s)$ has a holomorphic continuation. The Hurwitz zeta function $\zeta_{\mathscr{I}}(s, z)$ is also holomorphic on this domain, by the above claim, and the result follows by Theorem 4.

(vii) The Dirichlet series $\zeta_{\mathscr{A}}(s)$ is holomorphic for $\operatorname{Re}(s)>1$, and can be written as $\zeta_{\mathscr{I}}(s)=(s-1)^{-1 / 2} Z(s)$, where $Z(s)$ is holomorphic in a domain of the form

$$
\operatorname{Re}(s)>1-\frac{A}{[\log (|\operatorname{Im}(s)|+2)]^{A}}
$$

(see [20, pp.61-62]). An analogous statement is true for $\zeta_{\mathscr{I}}(s, z)$, by the above claim, and the result then follows by Theorem 4.

(viii) Clearly $\theta_{\mathscr{I}}=0$. Write $s=i t$, where $t \in \mathbb{R}$. We then have the formal expression

$$
\zeta_{\mathscr{I}}(i t)=\sum_{k=1}^{\infty} \xi^{\log n_{k}},
$$

where $\xi=e^{-i t}$. Now $n_{k+1} \geqslant n_{k}^{1+\gamma}$, so that $\log n_{k+1} \geqslant(1+\gamma) \log n_{k}$. A minor modification of Hadamard's gap theorem (see [48, § 7.43]) then implies that the circle $|\xi|=1$ is a natural boundary for the function of $\xi$ defined by (14). Therefore $\operatorname{Re}(s)=0$ is a natural boundary for $\xi_{\mathscr{I}}(s)$, and hence for $\zeta_{\mathscr{I}}(s, z)$. The result follows by Theorem 4 .

\section{References}

1. G. R. Allan, 'A spectral theory for locally convex algebras', Proc. London Math. Soc. (3) 15 (1965) 399-421.

2. T. Apostol, Introduction to analytic number theory (Springer, New York, 1976).

3. K. Babenko, 'On a problem of Gauss', Dokl. Akad. Nauk SSSR 238 (1978) 1021-1024 (Russian), Soviet Math. Dokl. 19 (1978) 136-140 (English).

4. O. F. BAndtLow, 'Spectral analysis of dynamical systems', PhD thesis, Cambridge University, 1997, http://www.maths.qmul.ac.uk/ ob.

5. O. F. BANDTLOW, 'Fine-structure of the spectra of intertwined operators', manuscript, Queen Mary, University of London, 2001.

6. C. Berenstein and R. GaY, Complex variables, an introduction (Springer, New York, 1991).

7. R. Bowen, 'Hausdorff dimension of quasi-circles', Inst. Hautes Études Sci. Publ. Math. 50 (1979) $11-25$.

8. R. T. Bumby, 'Hausdorff dimensions of Cantor sets', J. Reine Angew. Math. 331 (1982) 192-206.

9. U. Bunke and M. Olbrich, Selberg zeta and theta functions, a differential operator approach (Akademie Verlag, Berlin, 1995). 
10. P. Cartier and A. Voros, 'Une nouvelle interprétation de la formule des traces de Selberg', The Grothendieck Festschrift: a collection of articles written in honor of the 60th birthday of Alexander Grothendieck (ed. P. Cartier et al.), Progress in Mathematics 87 (Birkhäuser, Boston, 1990) 1-68.

11. F. Christiansen, P. Cvitanović and H.-H. Rugh, 'The spectrum of the period-doubling operator in terms of cycles', J. Phys. A 23 (1990) L713S-L717S.

12. C. Cowen and B. MACCLuer, Composition operators on spaces of analytic functions (CRC Press, Boca Raton, 1995).

13. T. W. Cusick and M. E. Flahive, The Markoff and Lagrange spectra, Mathematical Surveys and Monographs 30 (American Mathematical Society, Providence, RI, 1989).

14. H. Daudé, P. Flajolet and B. Vallée, 'An average-case analysis of the Gaussian algorithm for lattice reduction', Combin. Probab. Comput. 6 (1997) 397-433.

15. N. Dunford and J. T. Schwartz, Linear operators. Part I: general theory (Interscience, New York, 1957).

16. I. J. Good, 'The fractional dimension of continued fractions', Proc. Cambridge Philos. Soc. 37 (1941) 199-228.

17. S. Grabiner, 'Spectral consequences of the existence of intertwining operators', Ann. Soc. Math. Polonae 22 (1970) 227-238.

18. A. Grothendieck, Produits tensoriels topologiques et espaces nucléaires, Memoirs of the American Mathematical Society 16 (AMS, Providence, RI, 1955).

19. A. Grothendieck, 'La théorie de Fredholm', Bull. Soc. Math. France 84 (1956) 319-384.

20. G. H. Hardy, Ramanujan, 3rd edn (Chelsea, New York, 1978).

21. S. Heinemann and M. Urbański, 'Hausdorff dimension estimates for infinite conformal iterated function systems', Nonlinearity 15 (2002) 727-734.

22. D. Hensley, 'The Hausdorff dimensions of some continued fraction Cantor sets', J. Number Theory 33 (1989) 182-198.

23. E. Hille, Analytic function theory, Vol. II (Ginn, Boston, 1962).

24. O. Jenkinson and M. Pollicott, 'Computing the dimension of dynamically defined sets: $E_{2}$ and bounded continued fractions', Ergodic Theory Dynam. Systems 21 (2001) 1429-1445.

25. J. Jorgenson and S. LANG, Basic analysis of regularized series and products, Lecture Notes in Mathematics 1564 (Springer, New York, 1993).

26. J. JoRgENSON and S. LANG, Explicit formulas, Lecture Notes in Mathematics 1593 (Springer, New York, 1994).

27. H. Kamowitz, 'The spectra of endomorphisms of the disc algebra', Pacific J. Math. 46 (1973) 433-440.

28. E. Landau and A. Walfisz, 'Uber die Nichtfortsetzbarkeit einiger durch dirichletsche Reihen definierter Funktionen', Rendi. Circ. Mat. Palermo 44 (1919) 82-86.

29. G. Levin, 'On Mayer's conjecture and zeros of entire functions', Ergodic Theory Dynam. Systems 14 (1994) 565-574.

30. R. D. Mauldin and M. URbański, 'Dimensions and measures in infinite iterated function systems', Proc. London Math. Soc. (3) 73 (1996) 105-154.

31. R. D. Mauldin and M. URBaŃSKI, 'Conformal iterated function systems with applications to the geometry of continued fractions', Trans. Amer. Math. Soc. 351 (1999) 4995-5025.

32. D. H. MAYER, 'On composition operators on Banach spaces of holomorphic functions', J. Funct. Anal. 35 (1980) 191-206.

33. D. H. Mayer, 'On the thermodynamic formalism for the Gauss map', Comm. Math. Phys. 130 (1990) 311-333.

34. D. H. Mayer and G. RoepstorfF, 'On the relaxation time of Gauss' continued-fraction map. I: the Hilbert space approach (Koopmanism)', J. Statist. Phys. 47 (1987) 149-171.

35. D. H. MAYER and G. RoEPSTORFF, 'On the relaxation time of Gauss' continued-fraction map. II: the Banach space approach (transfer operator method)', J. Statist. Phys. 50 (1988) 331-344.

36. H. Mueller, 'Uber die meromorphe Fortsetzung einer Klasse verallgemeinter Zetafunktionen', Arch. Math. 58 (1992) 265-275.

37. S. Rolewicz, Metric linear spaces (PWN - Polish Scientific Publishers, Warsaw, 1972).

38. D. Ruelle, 'Zeta functions for expanding maps and Anosov flows', Invent. Math. 34 (1976) $231-242$.

39. D. Ruelle, Thermodynamic formalism (Wiley, New York, 1978).

40. D. Ruelle, 'Resonances of chaotic dynamical systems', Phys. Rev. Lett. 56 (1986) 405-407.

41. D. Ruelle, 'Resonances for Axiom A flows', J. Differential Geom. 25 (1987) 117-137.

42. D. Ruelle, 'An extension of the theory of Fredholm determinants', Inst. Hautes Études Sci. Publ. Math. 72 (1990) 175-193.

43. H.-H. Rugh, 'On the asymptotic form and the reality of spectra of Perron-Frobenius operators', Nonlinearity 7 (1994) 1055-1066.

44. J. Shallit, 'Real numbers with bounded partial quotients: a survey', Enseign. Math. 38 (1992) $151-187$ 
45. B. Simon, Trace ideals and their applications, London Mathematical Society Lecture Note Series 35 (Cambridge University Press, 1979).

46. A. E. TAYLOR, An introduction to functional analysis (John Wiley, New York, 1964).

47. S. Thangavelu, Lectures on Hermite and Laguerre expansions (Princeton University Press, 1993).

48. E. C. Titchmarsh, The theory of functions, 2nd edn (Oxford University Press, 1939).

49. A. Voros, 'Spectral functions, special functions, and the Selberg zeta function', Comm. Math. Phys. 110 (1987) 439-465.

50. G. N. Watson, A treatise on the theory of Bessel functions (Cambridge University Press, 1922).

51. E. Wirsing, 'On the theorem of Gauss-Kuzmin-Levy and a Frobenius type theorem for function spaces', Acta Arith. 24 (1974) 507-528.

Oliver Jenkinson

School of Mathematical Sciences

Queen Mary

University of London

Mile End Road

London E1 4NS

omj@maths.qmul.ac.uk

http://www.maths.qmul.ac.uk/ omj

Mariusz Urbański

Department of Mathematics

University of North Texas

Denton

TX 76203-1430

USA

urbanski@unt.edu

http://www.math.unt.edu/ urbanski
Luis Felipe Gonzalez

Mathematics Institute

University of Warwick

Coventry CV4 7AL

and

Department of Mathematics

University of North Texas

Denton

TX 76203-1430

USA

lfgonz@unt.edu 